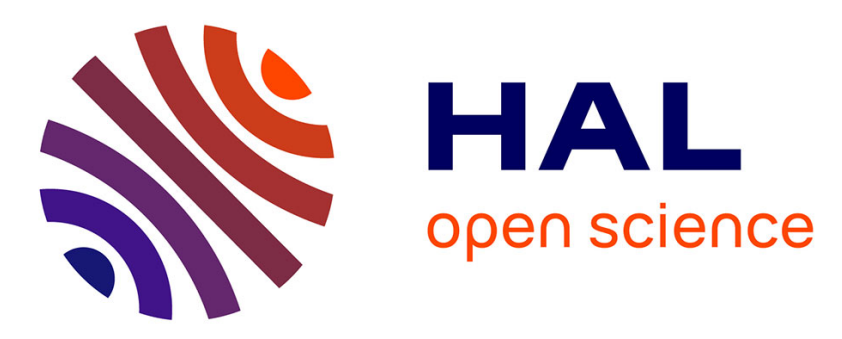

\title{
Effect of responsive graft length on mechanical toughening and transparency in microphase-separated hydrogels
}

Cécile Mussault, H. Guo, Nicolas Sanson, Dominique Hourdet, Alba Marcellan

\section{- To cite this version:}

Cécile Mussault, H. Guo, Nicolas Sanson, Dominique Hourdet, Alba Marcellan. Effect of responsive graft length on mechanical toughening and transparency in microphase-separated hydrogels. Soft Matter, 2019, 15 (43), pp.8653-8666. 10.1039/c9sm01178a . hal-02398343

\section{HAL Id: hal-02398343 \\ https://hal.science/hal-02398343}

Submitted on 10 Dec 2019

HAL is a multi-disciplinary open access archive for the deposit and dissemination of scientific research documents, whether they are published or not. The documents may come from teaching and research institutions in France or abroad, or from public or private research centers.
L'archive ouverte pluridisciplinaire HAL, est destinée au dépôt et à la diffusion de documents scientifiques de niveau recherche, publiés ou non, émanant des établissements d'enseignement et de recherche français ou étrangers, des laboratoires publics ou privés. 


\title{
Effect of responsive graft length on mechanical toughening and transparency in microphase-separated hydrogels
}

\author{
Cécile Mussault, Hui Guo, Nicolas Sanson, Dominique Hourdet, Alba Marcellan \\ alba.marcellan@espci.fr
}

Soft Matter Sciences and Engineering, ESPCI Paris, PSL University, Sorbonne University, CNRS, F-75005 Paris, France

\begin{abstract}
Effective remote control of mechanical toughening can be achieved by using thermoresponsive grafts such as poly ( $N$-isopropylacrylamide) (PNIPAm) in a hydrophilic covalently cross-linked polymer network. The weight ratio of PNIPAm grafts in the network may impart to such a thermo-responsive mechanical reinforcement. Here, we show that the network topology - especially graft length - is likewise crucial. A series of covalently cross-linked poly $(N, N$-dimethylacrylamide) (PDMA) gels grafted with PNIPAm side-chains of different lengths were designed and studied on both side of phase separation temperature $T_{c}$, at a fixed overall polymer concentration of $16.3 \mathrm{wt} \%$ and constant PDMA/PNIPAm weight ratio. Phaseseparated PNIPAm organic micro-domains were expected to act as responsive fillers above $T_{c}$ and to generate a purely organic nanocomposites (NC). In contrast to conventional NC gels where dissipative processes take place at the solid nanoparticle/matrix interface, here dissipation originates from the disruption of the filler itself by the unravelling of the PNIPAm grafts embedded in collapsed domains. Results show that PNIPAm graft length is a key parameter to enhance - reversibly and on-demand - the mechanical response. The longer the graft is, the more the mechanical toughening is effective. Interestingly, for long PNIPAm grafts, above $T_{c}$, the hydrogels combine perfect transparency together with both increased

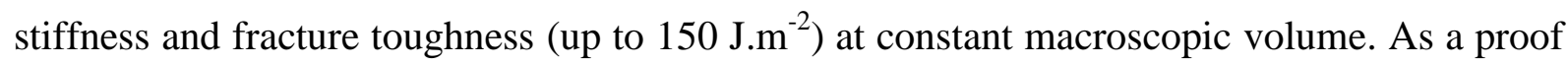
of concept, stimuli-responsive adhesion and shape-memory properties were designed to probe the inter-chain bridging efficiency (in bulk or bridging the interface).
\end{abstract}




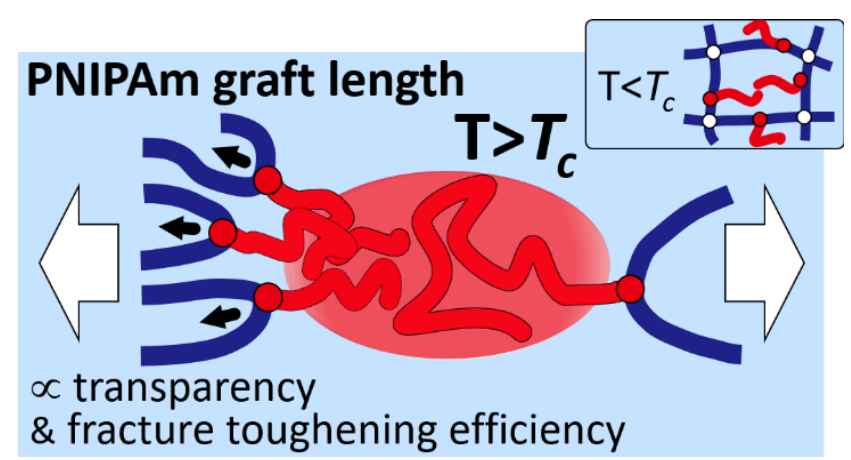

\section{Introduction}

The gel volume-phase transition ${ }^{1-8}$ has opened a world of possible functionalities as a new route to design active matter in wet conditions. Extremely large volume changes can thus be triggered by infinitesimal changes of environmental variables. This way, the thermoresponsiveness of poly( $N$-isopropylacrylamide) (PNIPAm) has been extensively investigated to design soft actuators in aqueous environments ${ }^{9}$ for fluidics ${ }^{10}$ or optics ${ }^{11}$, in the field of biomedical applications for drug delivery ${ }^{12}, 13$, for tissue engineering ${ }^{14,} 15$ or recently for information processing purposes. ${ }^{16}$ The thermo-responsiveness of PNIPAm is related ${ }^{6,7,17}$ to a sharp miscibility gap observed in aqueous solutions above its lower critical solution temperature (LCST), i.e., solutions of PNIPAm chains become hydrophobic and demix upon heating above $T_{c} \cong 31^{\circ} \mathrm{C}$. $^{7}$

In parallel, many efforts have been devoted to face the intrinsic fragility of covalently cross-linked gels by exploring a wide richness of macromolecular topologies. ${ }^{18,}{ }^{19}$ Based on the sacrificial bond concept, ${ }^{20}$ one promising strategy relies on introducing dissipative mechanisms in the network using either covalent or physical sacrificial bonds, as ionic bonds $^{21,22}$, hydrophobic interactions ${ }^{22-25}$ or using polymer adsorption onto nanoparticles in the case of nanocomposite hydrogels. ${ }^{26-29}$ More recently, stimuli-responsive gels have been developed using phase-separation as a new route of stimuli-responsive fracture toughening, ${ }^{30-}$ 32 dealing with the drastic change of polymer concentration entailed by the volume-phase transition. ${ }^{33,34}$

Our group designed original gel topologies that phase-separate under external stimuli at constant macroscopic volume (constant overall gel concentration). Among the various stimuli applicable, the thermo-responsiveness of PNIPAm is appealing because it can externally be applied in a non-invasive manner and performs reversibly. Hence, we demonstrated the remarkable asset that may represent the microphase-separation process for 
the design of gels displaying - on-demand and reversibly - advanced mechanical properties while maintaining the gel at constant and quite a high level of hydration: a 10-fold increase of the elastic modulus together with a 50-fold increase of the fracture energy (values up to 1000 J.m ${ }^{-2}$ with $83.3 \mathrm{wt} \%$ water) were achieved above $T_{c} .{ }^{33}$ The involved reinforcing mechanisms were attributed to stretch-induced dissipation by disruption of the phase-separated domains which act as reversible sacrificial links. It has been successfully demonstrated on poly $(N$ isopropylacrylamide) (PNIPAm) networks with covalently grafted $\operatorname{poly}(N, N$ dimethylacrylamide) (PDMA) side-chains that displayed extremely high fracture toughening with systematic crack bifurcation, as delamination in fiber-like composite materials. ${ }^{33}$ The network topology appeared to be key-aspect on fracture toughness: a cross-linked PNIPAm network grafted with PDMA side chains and a cross-linked PDMA network grafted with PNIPAm side chains performed differently. ${ }^{33}$ The substantial improvement in fracture resistance was attributed to the continuous structure generated by phase-separated domains in the case of cross-linked PNIPAm network with PDMA grafts ${ }^{35}$. This also opened the design of semi-interpenetrated architectures. ${ }^{36}$

In this framework, the most promising architecture - PNIPAm network with PDMA grafts - has been comprehensively investigated, but the study of the opposite topology remained incomplete. Questions remained open: Is it possible to improve the mechanical properties of the PDMA network by increasing the length of PNIPAm grafts, since optimal fracture toughening was achieved for continuous PNIPAm microphase separated domains? To what extent is this possible? For instance, by percolation of phase-separated domains into a PDMA network can an equivalent mechanical reinforcement be achieved? By using thermoresponsive grafts, the purpose of this work is to identify the relevant macromolecular parameters that allow for an effective mechanical toughening of the PDMA non-responsive network. Here, focus will be paid on the responsive-grafts, by varying in a systematic way their length and number, while studying the gels for identical polymer/water weight ratio on both side of the phase separation temperature.

As illustrated in Figure 1, the gel design relies on a hydrophilic covalently crosslinked $\operatorname{poly}(N, N$-dimethylacrylamide) (PDMA) network combined with responsive $\operatorname{poly}(N$ isopropylacrylamide) (PNIPAm) grafts acting as the thermo-sensitive reinforcing agent above $T_{c}$. The biphasic morphology of the grafted gels developed above $T_{c}$ implies the coexistence of swollen and collapsed domains, each domain differing in chain conformation, hydrophilicity/hydrophobicity and dynamics, but belonging to the same percolating hydrophilic network. We anticipate that the introduction of contrasting mechanical properties 
within the gel would produce mechanical reinforcement as in common nanocomposite (NC) gels by embedding inorganic nanofillers such as clay or silica. ${ }^{26-29,37}$ As illustrated in Figure 1, in contrast to $\mathrm{NC}$ gels where dissipative processes take place at the nanoparticle/matrix interface, here for $\mathrm{T}>T_{c}$, the mechanical dissipation is expected to originate from the disruption of the filler itself as described theoretically ${ }^{38}$ by unravelling of PNIPAm (hydrophobic) grafts into stretched chains. As in classical composite engineering, achieving stiffening together with fracture toughening is still challenging.

Varied controlled heterogeneous structures at $\mathrm{T}>T_{c}$, by changing the length and number of PNIPAm grafts, have been designed as model systems. The structure and macroscopic properties of these model networks have been investigated as a function of temperature by swelling experiments, differential scanning calorimetry, turbidimetry and small angle neutron scattering. The mechanical responses at small and large strains have been systematically studied under isochoric conditions on both side of the phase transition, i.e. keeping strictly constant the overall gel polymer concentration. This comprehensive set of analyses clearly highlights the determining role of network topology on the mechanical response, and more especially the significant impact of the responsive-graft length. For sufficient long grafts at $\mathrm{T}>T_{c}$, the hydrogels have demonstrated a quite remarkable combination of thermo-responsive properties: both increased stiffness and fracture toughness, enhanced adhesiveness together with perfect transparency above $T_{c}$. 


\section{EXPERIMENTAL SECTION}

Materials. $\quad N$-isopropylacrylamide (NIPAm, Aldrich), N,N-dimethylacrylamide (DMA, Aldrich), N,N'-methylenebisacrylamide (MBA, Aldrich), potassium peroxodisulfate (KPS, Aldrich) and $N, N, N^{\prime}, N^{\prime}$-tetramethylethylenediamine (TEMED, Aldrich) were used as received. Water was purified with a Millipore system combining inverse osmosis membrane (Milli RO) and ion exchange resins (Milli Q) for synthesis and purification.

Gel preparation. The synthesis of hydrogels was carried out in an ice bath under $\mathrm{N}_{2}$ atmosphere. PNIPAm macromonomers were prepared according to a standard method reported previously, ${ }^{39}$ with the molar mass controlled by adjusting the telogen and initiator concentration (see details in Supplementary Experimental data). Typically for GPD-Nx hydrogel: PNIPAm macromonomer $(1 \mathrm{~g}, 0.01 \mathrm{mmol})$, DMA monomer $(1 \mathrm{~g}, 8.8 \mathrm{mmol})$ and MBA crosslinker ( $1.36 \mathrm{mg}, 8.8 \mu \mathrm{mol}, 0.1 \mathrm{~mol} \%$ to DMA) were dissolved in a certain amount of water. After $30 \mathrm{~min}$ of deoxygenation with nitrogen bubbling, KPS (23.8 mg, $88 \mu \mathrm{mol}, 1$ mol\% to DMA), and TEMED (10.2 $\mathrm{mg}, 88 \mu \mathrm{mol}, 1 \mathrm{~mol} \%$ to DMA + PNIPAm) were dissolved separately, deoxygenated in water, and transferred subsequently into the aforementioned medium. The final solution was then rapidly transferred into syringes or between plates with a $2 \mathrm{~mm}$ gap under a nitrogen atmosphere. The reaction was left to proceed around $24 \mathrm{~h}$ at room temperature to fulfill the polymerization. Details of the formulations are given in Table $\mathbf{1}$.

SANS experiments. SANS experiments were performed at Laboratoire Léon Brillouin (CEA-Saclay, France) on the PAXY spectrometer. The wavelength of the incident neutron beam was set at $\lambda_{\theta}=15 \AA$ and $\lambda_{\theta}=6 \AA$ with a corresponding sample-to-detector distance of $3.0 \mathrm{~m}$ and $6.7 \mathrm{~m}$ respectively. These configurations provide a scattering vector modulus $\left[q=4 \pi \sin (\theta / 2) / \lambda_{\theta}\right]$ ranging between 0.002 and $0.2 \AA^{-1}$ (where $\theta$ is the scattering angle). For SANS experiments, gel plates of $2 \mathrm{~mm}$ thickness were specially synthesized in $\mathrm{D}_{2} \mathrm{O}$ to intensify the scattering contrast between the polymer network and the solvent. Gel discs (diameter $=14 \mathrm{~mm}$ and thickness $=2 \mathrm{~mm}$ ) punched from samples in their preparation state were fit inside a ring spacer hermetically sandwiched between two quartz slides. The gel samples were then placed in a temperature controlled auto-sampler and let to equilibrate at least during 1 hour at a given temperature (between 20 and $55^{\circ} \mathrm{C}$ ) prior to scattering experiments.

Differential Scanning Calorimetry (DSC). The phase transition was investigated by Differential Scanning Calorimetry using a DSC Q200 from TA instrument. Hydrogels at their 
preparation state (ca. $80 \mathrm{mg}$ ), equilibrated with a reference filled with the same quantity of pure water, were submitted to temperature cycles between 5 and $60{ }^{\circ} \mathrm{C}$ under nitrogen atmosphere. The heating and cooling rates were fixed at $10^{\circ} \mathrm{C} \cdot \mathrm{min}^{-1}$. Experiments were triplicate.

Transmittance $\boldsymbol{U} \boldsymbol{V}$-vis. Transmittance measurements were carried out at different temperatures with a UV-vis Hewlett-Packard 8453 spectrophotometer using a $0.2 \mathrm{~cm}$ path length quartz cell, and a wavelength of $520 \mathrm{~nm}$ and equipped with a heating/cooling rate of $0.25{ }^{\circ} \mathrm{C} \cdot \mathrm{min}^{-1}$. The hydrogel sample was directly synthesized in the UV-vis cell prior to the test.

Swelling measurements. Equilibrium swelling experiments were performed in pure water at designated temperatures. The samples, as prepared, were initially cut and placed in a large excess of water for one week. The swollen gels were weighed $\left(m_{t}\right)$ at equilibrium and the swelling ratio $(Q)$ were calculated as $Q=m_{t} / m_{d}, m_{d}$ being the dry weight measured after drying the swollen sample during two days at $60{ }^{\circ} \mathrm{C}$.

Rheology. The viscoelastic properties of hydrogels were studied in their preparation state, using a stress-controlled rheometer (DHR3 from TA Instruments) equipped with roughened plate/plate geometry (diameter $20 \mathrm{~mm}$, gap of $2 \mathrm{~mm}$ ). Particular care was taken to avoid the drying of the samples by immersing them into paraffin oil during the experiment. Both temperature and frequency sweep measurements were performed. The experiments were performed within the linear viscoelastic regime at $0.1 \%$ strain amplitude.

Temperature sweeps were performed at $1 \mathrm{rad} . \mathrm{s}^{-1}$ and a heating rate of $0.1{ }^{\circ} \mathrm{C} \cdot \mathrm{min}^{-1}$ as following: (1) heating from 25 to $60{ }^{\circ} \mathrm{C}$, (2) cooling until $10{ }^{\circ} \mathrm{C}$, (3) heating up to $60{ }^{\circ} \mathrm{C}$.

Frequency sweeps were performed at 20 and $60{ }^{\circ} \mathrm{C}$. Within these frequency tests, the angular frequency $\omega$ was varied from 1 to $100 \mathrm{rad} . \mathrm{s}^{-1}$.

Large strain behavior in tension mode. Tensile tests were performed on a standard tensile Instron machine, model 5565, equipped with a $10 \mathrm{~N}$ load cell (with a relative uncertainty of $0.16 \%$ in the range from 0 to $0.1 \mathrm{~N}$ ) and a video extensometer which follows the local strain at room temperature. The gels were then cut with final dimensions are $30 \mathrm{~mm} \times 5 \mathrm{~mm} \times 2$ $\mathrm{mm}$. The gauge length was taken constant $(\mathrm{L} 20 \mathrm{~mm})$ for all the tests. See Supplementary Figure S1 for geometry details.

For high temperatures or long-term experiments, in order to isolate the samples from the environment (prevent evaporation or swelling), an immersion cell was designed, consisting of a paraffin oil bath surrounding the sample and allowing a precise control of the temperature. 
Nominal stress was defined as $\sigma=F / S_{0}$, with $F$ being the recorded force and $S_{0}$, the initial cross section. Reduced stress, $\sigma_{R}$ was calculated as following: $\quad \sigma_{R}=\frac{\sigma}{\left(\lambda-\lambda^{-2}\right)}$

With $\sigma$ being the nominal stress and $\lambda$, the elongation which is defined as: $\lambda=L / L_{0}=1+\varepsilon$.

Gels were studied from their preparation state. Different types of mechanical tests were carried out at the designated temperature: monotonic tensile tests, loading-unloading cycles and fracture tests. All the tests were carried out at a nominal strain rate of $0.06 \mathrm{~s}^{-1}$.

Fracture tests. Fracture was studied using the single edge notch geometry. A notch, $c$ of approximately $1 \mathrm{~mm}$ length was made on the edge of a gel strip. The gauge length was fixed at $\mathrm{L} \sim 20 \mathrm{~mm}$. The fracture energy, $G_{c}$ was calculated using the following expression: ${ }^{40}$ $G_{c}=(6 \cdot W \cdot c) / \sqrt{ } \lambda_{c}$ with $c$ the initial notch length, $\lambda_{c}$ the strain at break and $W$ the strain energy density calculated by integration of the nominal stress-strain curve.

Adhesion tests. Single lap-shear geometry was used, see Supplementary Figure S1 for details of the geometry. The lap-shear assembly was made at room temperature under a contact pressure of $1 \mathrm{kPa}$ during $1 \mathrm{~min}$, then thermalized at $60{ }^{\circ} \mathrm{C}$ (immersed in oil) for $10 \mathrm{~min}$ before launching the test. The overlap length was $l=5 \mathrm{~mm}$, using the ribbon thickness and width of $2 \mathrm{~mm}$ and $5 \mathrm{~mm}$, respectively. The total length of the assembled ribbons was $40 \mathrm{~mm}$. When failure occurred by interfacial peeling, the adhesion energy was estimated from the adhesive failure force $F$ as follows: ${ }^{41} G_{a d h}=3(F / w)^{2} /(2 E h)$, where $w$ and $h$ denote, respectively, the width and thickness of the ribbon, and $E$ is the tensile modulus.

\section{RESULTS AND DISCUSSION}

\section{Gel synthesis and formulation}

The synthesis of PDMA networks by free radical polymerization was shown to be simple and robust. ${ }^{29,42} 43$ This method allowed the preparation of networks with grafted side-chains within a quite broad range of formulation parameters as described elsewhere. ${ }^{33,39}$ The GPD-N topologies (see Figure 1 and Table 1) were prepared by in situ free radical crosslinking polymerization in water of DMA monomer, MBA crosslinker in presence of PNIPAm macromonomers using TEMED and KPS as co-initiators. GPD-N refers to PDMA (D) networks with grafted PNIPAm (N) macromonomers. All gels formulations were prepared at a fixed weight fraction of polymer, $w_{0}$, of $16.7 \mathrm{wt} \%$ (83.3 wt $\%$ hydration level). This corresponds to a swelling degree at preparation state, $Q_{0}=6$, the swelling degree being defined as $Q=1 / w$, with $w$ referring to the polymer mass fraction. Networks were prepared 
preserving a constant crosslinker/monomer ratio, $R=(\mathrm{MBA}) /(\mathrm{DMA})=0.1 \mathrm{~mol} \%$. This small value of chemical crosslinker was a compromise to overcome the self-crosslinking process that takes place during DMA polymerization ${ }^{42}$ and to avoid dramatic reduction of network extensibility. This $R=0.1 \mathrm{~mol} \%$ ratio corresponds to a theoretical number of DMA monomer units between crosslinks $N=500$, since $N$ is given by $N=2\left(\mathrm{M}_{0}\right) / f \cdot\left(\mathrm{X}_{0}\right)=2 / f \cdot R$ with $\left(\mathrm{M}_{0}\right)$ and $\left(\mathrm{X}_{0}\right)$ being the number concentration of monomer (DMA) and cross-linker (MBA), respectively and $f$ refers to the cross-linker functionality (here 4 ).

The GPD-N $\boldsymbol{x}$ series refers to networks with varied graft lengths of PNIPAm macromonomers, in which $x$ stands for the average molar mass of PNIPAm grafts in kg.mol ${ }^{-1}$. The weight ratio between the PDMA network and the PNIPAm grafts was kept constant at 50/50 to balance the hydrophilic/hydrophobic contributions and impede a macroscopic collapse of the gel at $\mathrm{T}>T_{c}$. As reported in Table 1 and Supplementary Figure S2, the average molar masses of PNIPAm grafts were varied from 20 to $120 \mathrm{~kg} \cdot \mathrm{mol}^{-1}$, i.e. about 170 to 1000 NIPAm monomer units. Note that in this series the weight content of PNIPAm responsive grafts is strictly identical (8.35 wt\%).

Another series, denoted as GPD-N55 (y/z) was designed as a control series. In this case, the average molar mass of PNIPAm macromonomers grafted to the PDMA network was kept at $55 \mathrm{~kg} \cdot \mathrm{mol}^{-1}$. While the overall polymer weight ratio was kept constant, (i.e., $w_{0}=16.7$ wt $\%$ ), the PDMA/PNIPAm weight ratios were varied from 69/31 to 31/69. With the same overall polymer concentration, the idea was to study the effect of the relative amount of PNIPAm grafts acting as fillers by analogy with NC gels. This implies a PDMA network weight fraction in the gel varying from 11.5 to $5.2 \mathrm{wt} \%$, respectively. The crosslinker ratio $R$ was again fixed at $0.1 \mathrm{~mol} \%$.

All these formulations, after full completion of the polymerization (24h), gave rise to homogeneous, transparent and elastic gels at room temperature. Extractables were measured after swelling and ranged from 5 to $10 \mathrm{wt} \%$ of initial polymer content, confirming the good reliability of the polymerization process, taking into account that extractables mainly arise from the polymerization of the PDMA network in water at $8.35 \mathrm{wt} \%$ and, to a lesser extent, from some ungrafted macromonomers (see Table 1).

Note that GPD-N55(31/69) gels appeared to be quite weak and sticky when remove from their molds given the low concentration of network, close the gel point. 


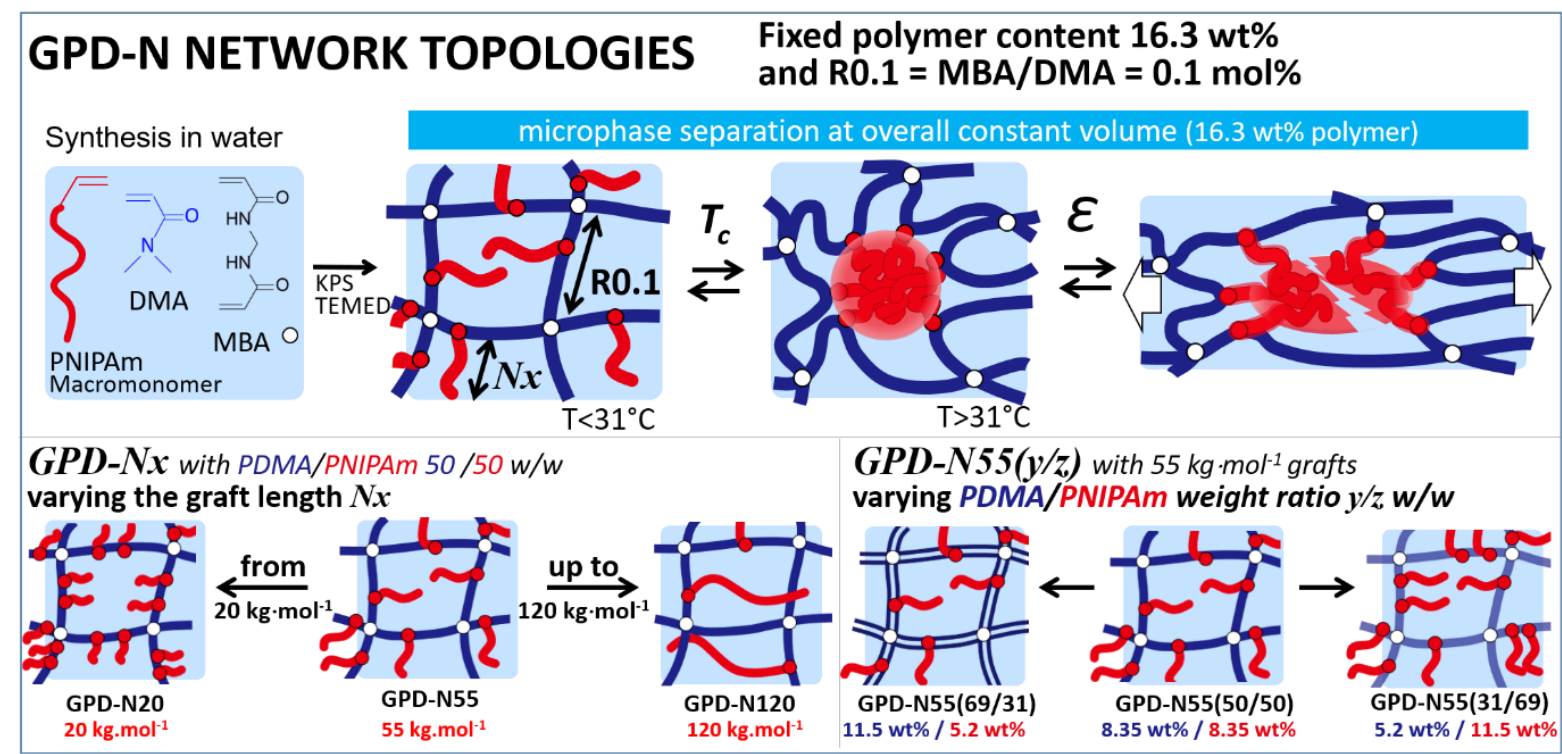

Figure 1. Schematic representation of GPD-N gel topologies. Thermo-associative PNIPAm chains are depicted in red and non-responsive PDMA chains in blue. All gels formulations were defined and studied at a fixed weight fraction of polymer, $w_{0}$, of $16.7 \mathrm{wt} \%$ together with a fixed crosslinker/monomer ratio, $R$, of $0.1 \mathrm{~mol} \%$. Two series were investigated. The GPD$\mathrm{Nx}$ series was designed with an equal weight fraction of PNIPAm macromonomer and PDMA network 50/50 w/w and various graft lengths. The GPD-N55(y/z) series holds the grafted networks prepared with the same PNIPAm macromonomer $\left(x=55 \mathrm{~kg} . \mathrm{mol}^{-1}\right)$ but various weight ratio $(y / z)$ between PDMA and PNIPAm.

\begin{tabular}{|c|c|c|c|c|c|c|c|c|c|c|c|}
\hline \multirow[b]{2}{*}{ Sample } & \multirow{2}{*}{$\begin{array}{l}\text { Water } \\
(\mathrm{g})\end{array}$} & \multirow{2}{*}{$\begin{array}{l}\text { DMA } \\
(\mathrm{g})\end{array}$} & \multicolumn{4}{|c|}{ Macromonomer-PNIPAm } & \multirow[t]{2}{*}{$Q_{0}$} & \multirow{2}{*}{$\begin{array}{c}\text { Extractables } \\
\quad(\text { wt \%) }\end{array}$} & \multirow{2}{*}{$\begin{array}{c}\Delta H \\
\left(\mathrm{~kJ} \cdot \mathrm{mol}^{-1 *}\right) \\
{ }^{*} \text { NIPAm }\end{array}$} & \multirow{2}{*}{$\begin{array}{c}Q_{\mathrm{eq}} \\
\mathrm{T}=20^{\circ} \mathrm{C}\end{array}$} & \multirow{2}{*}{$\underset{\mathrm{T}=600^{\circ} \mathrm{C}}{Q_{\mathrm{eq}}}$} \\
\hline & & & $\begin{array}{l}\text { PNIPAm } \\
(\mathrm{g})\end{array}$ & $\begin{array}{c}M_{\mathrm{n}} \\
\left(\mathrm{kg} \cdot \mathrm{mol}^{-1}\right)\end{array}$ & $D P_{\mathrm{n}}$ & 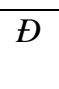 & & & & & \\
\hline homo-PDMA & 9.14 & 0.83 & 0 & -- & -- & -- & 12 & $12.3 \pm 0.5$ & -- & $69.4 \pm 0.6$ & $52.3 \pm 5.0$ \\
\hline GPD-N20 & 8.31 & 0.83 & 0.83 & 18.9 & 177 & 1.2 & 6 & $10.3 \pm 0.1$ & $4.0 \pm 0.2$ & $37.7 \pm 0.6$ & $17.1 \pm 0.6$ \\
\hline GPD-N30 & 8.31 & 0.83 & 0.83 & 32.6 & 303 & 1.5 & 6 & $11.4 \pm 0.1$ & $4.7 \pm 0.2$ & $36.7 \pm 0.6$ & $13.8 \pm 0.6$ \\
\hline GPD-N40 & 8.31 & 0.83 & 0.83 & 39.3 & 354 & 1.3 & 6 & $11.2 \pm 1.5$ & $3.7 \pm 0.2$ & $49.1 \pm 0.6$ & $9.9 \pm 0.6$ \\
\hline GPD-N55 & 8.31 & 0.83 & 0.83 & 54.9 & 488 & 1.4 & 6 & $9.4 \pm 2.0$ & $3.4 \pm 0.2$ & $38.7 \pm 2.4$ & $10.4 \pm 0.6$ \\
\hline GPD-N90 & 8.31 & 0.83 & 0.83 & 87.2 & 798 & 1.4 & 6 & $6.2 \pm 1.0$ & $4.8 \pm 0.5$ & $44.1 \pm 1.2$ & $9.9 \pm 1.2$ \\
\hline GPD-N120 & 8.31 & 0.83 & 0.83 & 121.0 & 1061 & 1.5 & 6 & $5.5 \pm 0.6$ & $3.8 \pm 0.2$ & $41.2 \pm 0.6$ & $9.4 \pm 0.6$ \\
\hline GPD-N55(69/31) & 8.30 & 1.15 & 0.52 & 54.9 & 488 & 1.4 & 6 & $4.8 \pm 1.6$ & $4.2 \pm 0.2$ & $35.3 \pm 1.8$ & $16.6 \pm 0.2$ \\
\hline GPD-N55(31/69) & 8.32 & 0.52 & 1.15 & 54.9 & 488 & 1.4 & 6 & $17.1 \pm 3.0$ & $3.9 \pm 0.1$ & $68.0 \pm 1.8$ & $6.9 \pm 1.3$ \\
\hline
\end{tabular}

Table 1. Formulations of GPD-Nx and GPD-N55(y/z) hydrogels. PNIPAm macromonomers were characterized by Size Exclusion Chromatography: number-average molar mass, $M_{n}$, degree of polymerization, $D P_{n}$, and dispersity, $Ð$, are reported. $\Delta H$ is the enthalpy obtained involved at phase transition obtained by Differential Scanning Calorimetry. $Q_{0}$ and $Q_{e q}$ are respectively the swelling ratio at the preparation state and at thermodynamic equilibrium in water. Extractables were obtained after swelling. 


\section{Micro-phase separation temperature and structural heterogeneities}
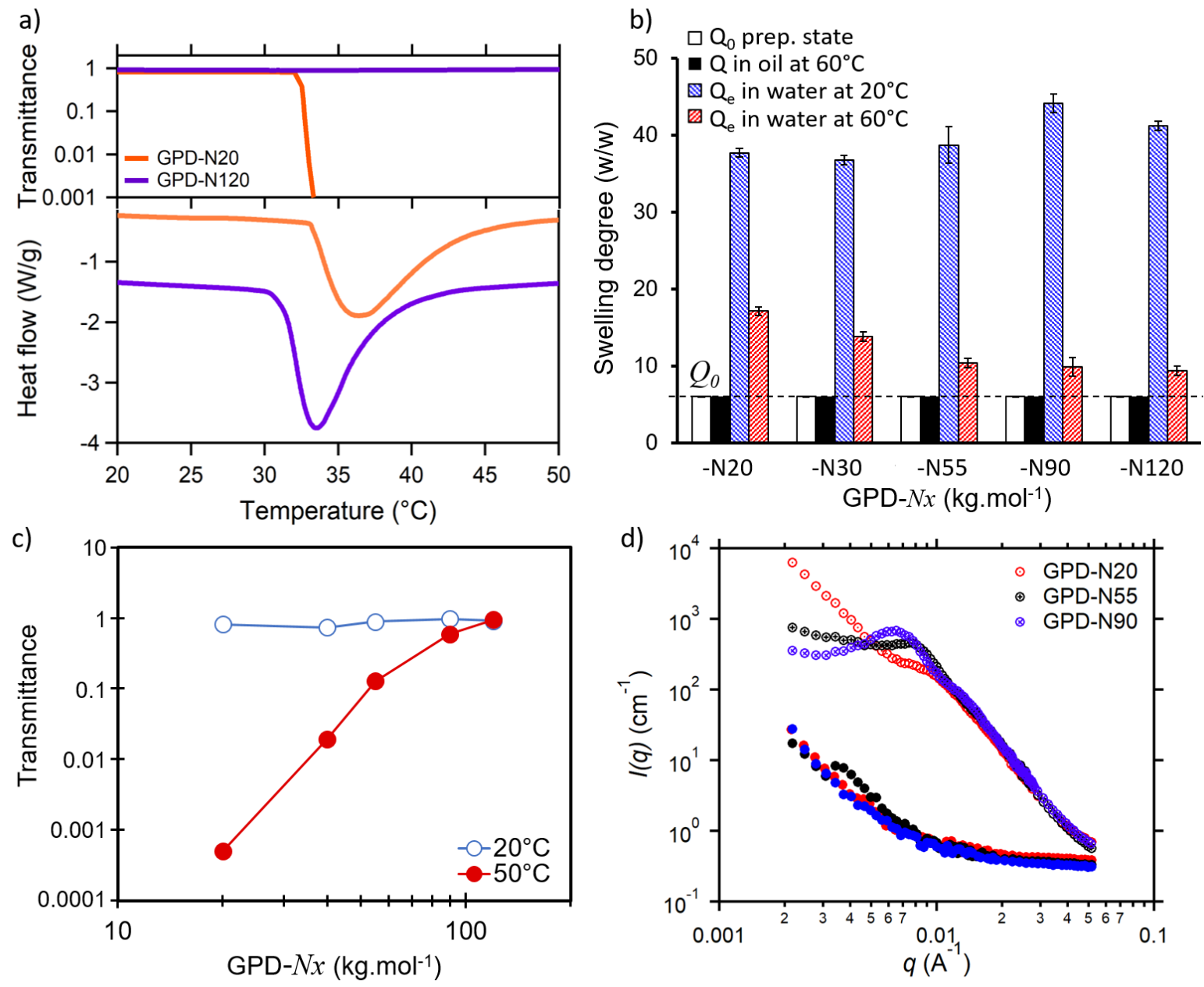

Figure 2. Phase-transition of PNIPAm grafts and swelling behavior of GPD-Nx gels. a), Endotherms obtained for GPD-N20 and GPD-N120 by differential scanning calorimetry upon heating reflects the phase-transition undergone by PNIPAm side-chains forming microdomains. In insert the corresponding transmittance. b), Swelling degree at preparation conditions and at equilibrium in water at $20{ }^{\circ} \mathrm{C}$ and $60{ }^{\circ} \mathrm{C}$. Note that gel composition can be easily fixed at its preparation state on both sides of the phase transition by immersing it in paraffin oil. Here, gels at preparation state were immersed in paraffin oil, swelling was measured for 1 day at $60{ }^{\circ} \mathrm{C}$. c), Transmittance of GPD-Nx series at $20{ }^{\circ} \mathrm{C}$ (preparation state) and at $50{ }^{\circ} \mathrm{C}$ (phase-separated state). Abscissa refers to the molar mass of the grafted PNIPAm side chains. d), Scattering data of GPD-N20, GDD-N55 and GPD-N90 obtained at $20{ }^{\circ} \mathrm{C}$ (filled symbols) and $55^{\circ} \mathrm{C}$ (hollow symbols).

As expected from the LCST-type phase transition of PNIPAm in aqueous solutions, all networks featured an endothermic transition upon heating reflecting the coil-to-globule conformational change of PNIPAm side-chains, as illustrated in Figure 2a for GPN20 and GPN-120. The related transition enthalpies, around $4 \mathrm{~kJ}$ per mole of NIPAm for all the series (see Table 1 and Supplementary Figure S3), state the disruption and reorganization of water 
molecules initially solvating NIPAm units by strong hydrogen bonding along with the hydrophobic interactions between isopropyl groups that induce the collapse of the chain. ${ }^{44-46}$ Despite some differences in $T_{c}\left(T_{c}\right.$ varies from 32 to $\left.36{ }^{\circ} \mathrm{C}\right)$ or peak broadening, no clear detectable trend was noticed by calorimetry on the effect of graft length for the GPD-Nx series. This result seems consistent with both experimental and theoretical work reported for PNIPAm solutions, especially for chains with DP $>100 .^{7,47}$

By immersing the gel in pure water from its preparation state $\left(Q_{0}=6\right)$, as expected, the GPD$\mathrm{Nx}$ gels at $20{ }^{\circ} \mathrm{C}$ swell up to an equilibrium value, $Q_{e}$, that is around 5-fold with respect to their initial swelling at preparation state due to the hydrophilic nature of both the PDMA network and the PNIPAm grafts below their LCST (see Figure 2 and Table 1). Above $T_{c}$, PNIPAm phase separates and GPD-Nx gels sharply deswell with respect their equilibrium state in water at $20{ }^{\circ} \mathrm{C}$. The length of PNIPAm side chains seems to contribute positively for gel deswelling. This suggest that hydrophobic side-chains are capable of interconnecting to impose the deswelling of the PDMA network. It is noteworthy that in water at $60{ }^{\circ} \mathrm{C}$, despite the microphase separation, GPD-Nx gels sustain almost their initial swelling, or slightly swell from $Q_{0}$. Since water is not squeezed out from the gel in such conditions, the gel composition can be easily fixed at preparation state on both sides of the phase transition by immersing it in paraffin oil. This method has been previously used in our systems. ${ }^{29,33,42}$ As shown in Figure $\mathbf{2 b}$, no evidence of any interactions between the paraffin oil and the gel or composition drifts at $60{ }^{\circ} \mathrm{C}$ overtime ( 1 day) were noticed. Note that a pure homo-PNIPAm network would reach an equilibrium swelling at $60{ }^{\circ} \mathrm{C}$ of about 2.3 .

While the micro-phase separation proceeds and involves almost identical enthalpy for the GPD-Nx series, suggesting that all the PNIPAm chains exhibit an equivalent level of dehydration whatever their length, strong differences on gel turbidity were observed. Some gels appeared opaque and white, while others seemed to do not change aspect. As reported in Figure 2c, at $50{ }^{\circ} \mathrm{C}$ GPD-Nx gels displayed a sharp transmittance increase with the rise of the PNIPAm graft size. Surprisingly, GPD-N90 and GPD-N120 hydrogels phase-separate without any visible change to the naked eye compared to their preparation state. Such a feature highlights strong differences in structural heterogeneities in the gel network. This is reasonable to assume from DSC results that the relative optical indexes of the phase-separated domains with respect to the medium are almost the same for all the GPD-Nx series, and additionally, gels hold the same concentration of phase separated domains. This sharp optical 
signature of GPD-Nx gels, from perfectly transparent to opaque, seems to indicate differences in the sizes of the phase separated domains.

Complementary experiments were carried out by small angle neutron scattering (SANS), below and above the transition temperature (20 and $55{ }^{\circ} \mathrm{C}$, respectively) in order to investigate the nanostructure formation of the polymer network. As seen in Figure 2d, the self-association of PNIPAm side-chains above $T_{c}$ gives rise to a huge increase of the scattering intensity with a correlation peak at $q_{\max }$ which underlines some characteristic distance between rich-PNIPAm domains. $\left(d \sim q_{\max }{ }^{-1}\right)$. As the position of $q_{\max }$ decreases slightly with the length of the graft, this means that the distance between the collapsed domains increases, as well as their size, since the number of domains decreases at a fixed PNIPAm content. In the high $q$-range and at high temperature, the scattered intensities decay following the Porod law, $I(q) \sim q^{-4}$, characteristic of a two-phases morphology with a sharp interface, here between rich-PNIPAm domains and the swollen PDMA matrix. Interestingly, all the scattering curves are superimposed in the high $q$-regime. which means that the morphology of PNIPAm domains within the PDMA network are rather similar whatever the length of PNIPAm side-chains. On the other hand, there are large differences of the scattering behavior at low $q\left(q \leq 4.10^{-3} \AA^{-1}\right)$ between GPD-Nx hydrogels with an increase in scattering intensity with decreasing the average molar mass of PNIPAm grafts. This intensity upturn observed at low $q$ is the signature of larger fluctuations revealing the presence of strong attractive interactions between phase-separated domains forming larger aggregates. This result is in good agreement with the turbidity experiments plotted in Figure $2 \mathbf{c}$ where it was shown that the absorbance at high temperature strongly increases when decreasing the molar mass of PNIPAm side-chains. This is especially the case for GPD-N20 which also exhibits a $q^{-4}$ dependence at low $q$ value evidencing the existence of larger aggregates with sharp interfaces. We anticipated this difference of morphology between grafted hydrogels to have an impact on their mechanical response.

\section{Thermoresponsiveness of GPD-Nx gels by Linear Rheology}

The mechanical properties of the thermoresponsive grafted networks were first studied at small deformations, probing the mechanical response of the microstructure without strong perturbations from its conformation at rest. Note that samples were studied from their preparation state under isolated conditions, i.e. immersed in paraffin oil, to prevent water evaporation or swelling. As already pointed out from the thermodynamic and structural 
viewpoint, the microphase-separation process also triggers dramatic changes in the viscoelastic properties near $T_{c}$. Such thermo-responsiveness is illustrated in Figure 3 with GPD-N55 gels studied at a constant frequency. By heating the sample from its preparation state above $T_{c}$, the emergence of collapsed domains within the gel gives rise to a rather sharp increase of both elastic and loss moduli, respectively $G^{\prime}$ and $G$ ". This stiffening reflects the composite structure of the gel which can be pictured as collapsed hydrophobic PNIPAm domains exhibiting reduced dynamics, ${ }^{39}$ embedded within a soft and highly hydrated PDMA matrix. This general feature, already reported for homo-PNIPAm networks was assigned to the emergence of heterogeneities in gel dynamics at phase separated state ${ }^{48-51}$. Note that sufficiently far from $T_{c}$, the contribution of dissipative losses remains quite low and almost the same since $G$ " $/ G^{\prime}$ is around 0.1 at 25 and $60{ }^{\circ} \mathrm{C}$, respectively.

As observed in Figure 3a and $\mathbf{3 b}$, the pristine properties are fully recovered when temperature is lowered below $T_{c}$, with almost no hysteresis when the heating/cooling rate is low enough (here $0.1{ }^{\circ} \mathrm{C} \cdot \mathrm{min}^{-1}$ ). Different cycles of heating/cooling showed perfectly superimposable behavior, demonstrating the high reproducibilty of the generated nanostructure. This perfect reversibility confirms that water is not expelled from the sample upon heating and during the timescale of the experiment (about tens of hours) but absorbed by the PDMA hydrophilic network.

As shown in Figure 3c, the same general trend was observed for the different lengths of grafted side chains. Note that $T c$ values obtained from the $G$ ' onset or $\tan \delta$ maximum rather good accordance with the previous calorimetric and turbidimetric observations (see Supplementary Figure S3). As summarized in Figure 3d, all the gels exhibit a significant enhancement of stiffness in the microphase separated regime. Nevertheless, while the overall polymer concentration and composition of the gels were strictly identical, the efficiency of the mechanical thermo-reinforcement varies significantly with the graft layout.

At $20{ }^{\circ} \mathrm{C}$, at the coil state, the elastic shear modulus reflects the topology of the grafted PDMA networks. The values of elastic shear moduli, $G^{\prime}$ measured at $20{ }^{\circ} \mathrm{C}$ are rather similar for the GPD-Nx series, around $0.9 \pm 0.3 \mathrm{kPa}$. Such a value is in line with the response of homo-PDMA network at $8.3 \mathrm{wt} \%\left(G^{\prime} \cong 1.0 \mathrm{kPa}\right)$, designed and synthesized as control experiment to be an analogous of GPD-Nx without grafts (see Supplementary Figure S4). This result suggests that GPD-Nx gels have an equivalent PDMA cross-linked network. On the basis of the entropy-driven elasticity theory in phantom network ${ }^{52,53}$, each elastically 
active chain of the network is supposed to contribute equally for $k T$ to the modulus. The average molar mass between effective cross-links can be calculated for the GPD-Nx series and gives an average value of about $120 \mathrm{~kg} \cdot \mathrm{mol}^{-1}$, i.e., a number of DMA monomer units between crosslinks of 1200. Accounting for measurement uncertainties, it seems that a slightly higher $G$ ' values are observed for GPN-D90 and GPN-D120, possibly due the contribution of the high length PNIPAm side chains to entanglements or to additional crosslinks related to transfer reactions during polymerization. ${ }^{54}$

a)

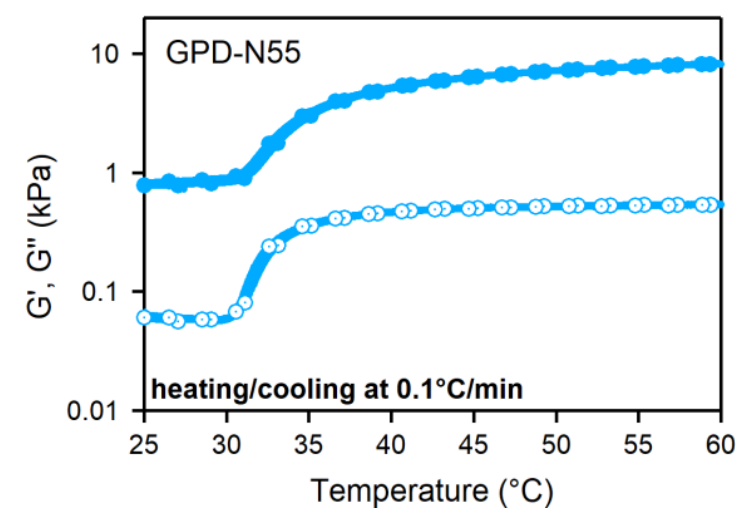

c)

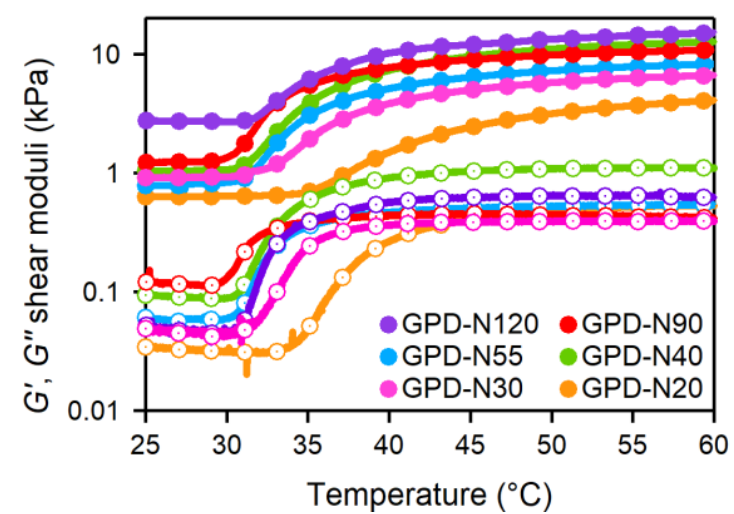

b)

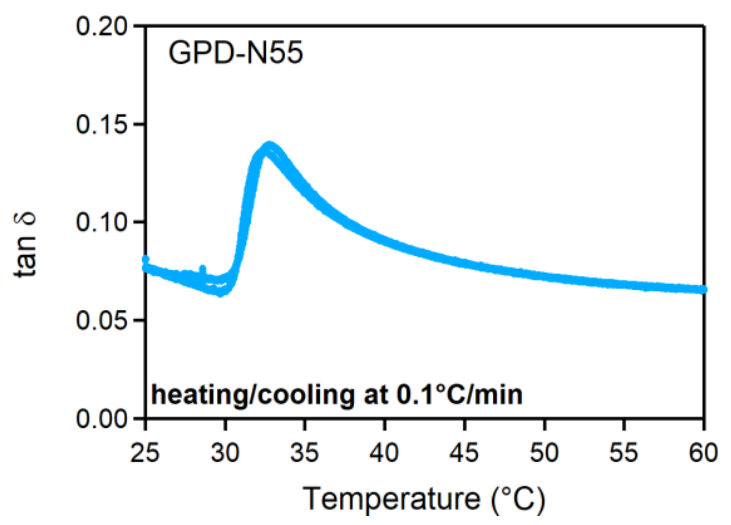

d)

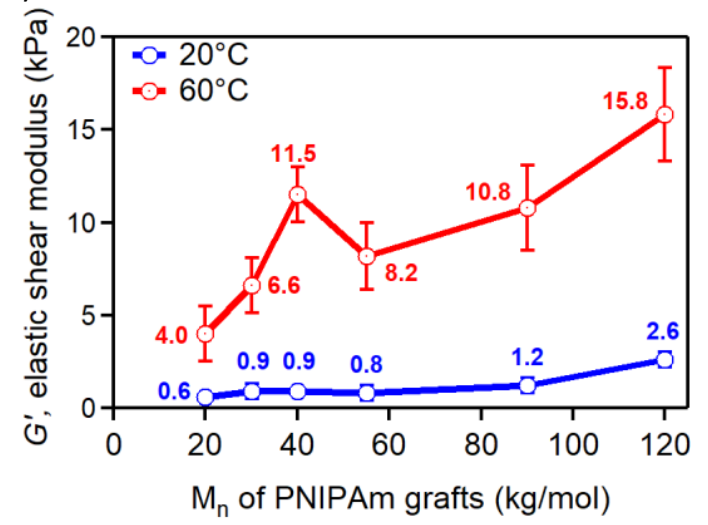

Figure 3. Viscoelastic behavior of the GPD-Nx series vs. Temperature. GPD-N55 gels upon thermal cycling (a) Shear storage modulus, $G$ ' (filled symbols) and loss modulus, $G$ '” (open symbols) and (b) the loss tangent, $\tan \delta$, for a heating and cooling rate of $0.1{ }^{\circ} \mathrm{C} \cdot \mathrm{min}^{-1}$. (c) Shear elastic and storage moduli for the GPD-Nx series. (d), Impact of graft length on the storage shear modulus $G$ ' at $20{ }^{\circ} \mathrm{C}$ (blue) and $60{ }^{\circ} \mathrm{C}$ (red).

\section{GPD-Nx gel dynamics by Linear Rheology}

The signature of topological defects in polymer networks, as grafted side chains, have been analyzed in light of the relaxation processes by scanning the shear frequency below $T_{c}$. Figure 4a compares the dynamics of GPD-Nx networks at $20{ }^{\circ} \mathrm{C}$ for two graft lengths (40 $\mathrm{kg} \cdot \mathrm{mol}^{-1}$ and $120 \mathrm{~kg} \cdot \mathrm{mol}^{-1}$ ) with the homo-PDMA network. The amplitude of the loss 
modulus, $G$ ', is directly related to the number of dangling chains and to their relative length with respect to the mesh size of the network. ${ }^{55}$ In the case of homo-PDMA gels, as expected, the $G$ " contribution is lower than for their GPD-Nx counterparts. However, the frequencydependence, $G$ "' $\sim \omega^{\alpha}$, observed for homo-PDMA gels (with $\alpha=0.5$ ), highlights the presence of defects in the network that have been frozen during the polymerization process. At a molecular level, the power-law relaxation process reflects the (slow) relaxation rate of dangling chains in the presence of topological constraints ascribed to the network. ${ }^{1}$ For a network with a given mesh size, $\xi, \alpha$ is expected to decrease with $l$, the length of the grafts ${ }^{56}$ due to an increase of constraints per dangling chain. This is confirmed for GPD-N40 and GPD-N120 gels, as shown in Figure 4a, the presence of the grafts slows down the relaxation dynamics, as $\alpha=0.4$ for GPD-N40 and GPD-N120 compared to $\alpha=0.5$ for the PDMA network without grafts. No significant effect of chain length was observed within the experimental range of $N x$ investigated, suggesting quite a broad distribution in the network mesh size, $\xi$ due to the random nature of the polymerization processes., ${ }^{1,57,58}$

As shown in Figure $4 \mathbf{b}$, at $60{ }^{\circ} \mathrm{C}$ the relaxation processes of GPD-Nx gels seem to be suppressed in the frequency range investigated since $G^{\prime}$ and $G^{\prime \prime}$ remain almost constant in contrast to the homo-PNIPAm gels. ${ }^{48}$ This highlights the stability of PNIPAM aggregates and the fact that they dominate the mechanical response of the gel within this regime of strain and frequency investigated.

a)

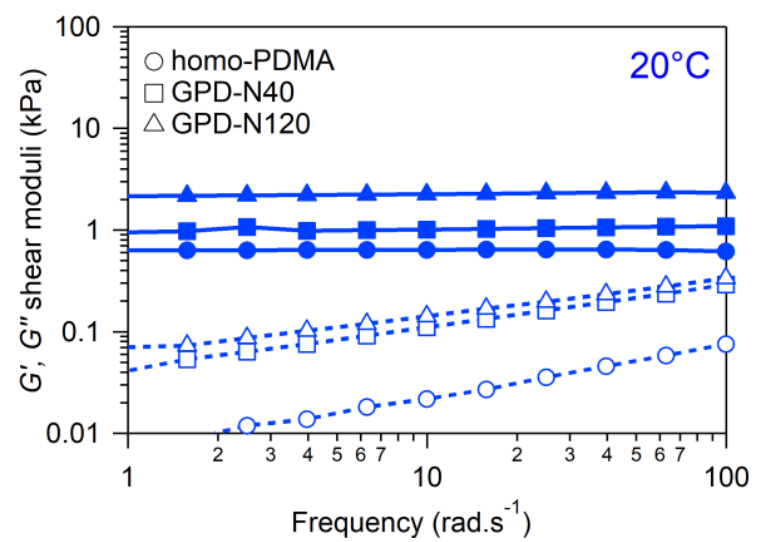

b)

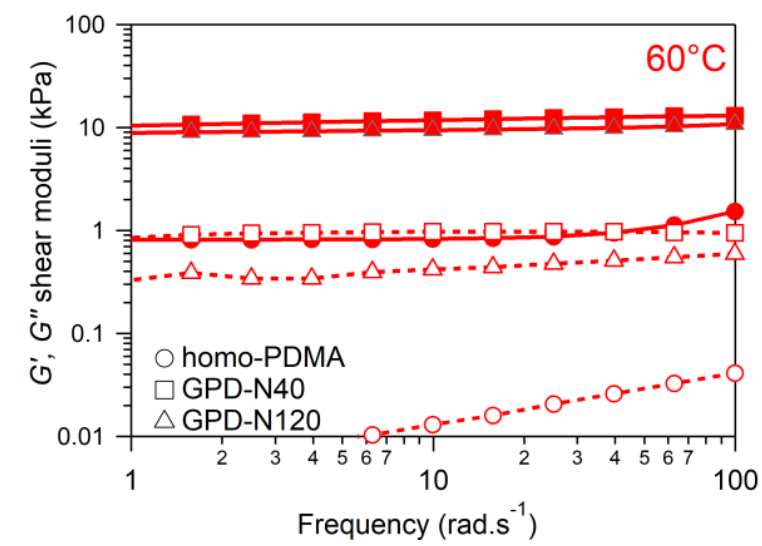

Figure 4. Frequency dependence of the storage (solid symbols, G') and loss (open symbols, G”) moduli of representative GPD-Nx hydrogels (with $x=0,40$ and 120) measured at (a) 20 ${ }^{\circ} \mathrm{C}$ and (b) $60{ }^{\circ} \mathrm{C}$.

\section{Large strain mechanical behavior and self-recovery}


Figure 5a and $\mathbf{5 b}$ shows the large strain behavior of GPN-D55 gels in tensile mode that has been investigated at 20 and $60{ }^{\circ} \mathrm{C}$, sufficiently far from the phase separation temperature. While linear rheology does not involve large structural perturbations, large strain experiments enable to explore the capabilities of the network to self-reorganize under stress. The PDMA network is supposed to feature entropy-driven elasticity on either side of the phase-separation temperature and to transmit stresses to the phase-separated domains since it is topologically defined as the load-bearing phase and being non-thermoresponsive. To graphically enhance the deviation from entropy-driven elasticity, stress-strain behaviors have been plotted as reduced stress, ${ }^{59} \sigma_{R}$ versus reciprocal elongation, $1 / \lambda$ to focus on the early stages of deformation. Commonly used for rubbers, this semi-empirical model enables one to separate the signature of the cross-links from the one of entanglements. The stretching of an ideal network made of Gaussian (without entanglements) chains between crosslinks is expected to give rise to a horizontal line. For unfilled rubbers, appreciable deviations from this idealized network have long been studied, ${ }^{60}$ demonstrating softening at low and moderate extensions, which are assigned to the presence of entanglements and their capability to facilitate conformational changes, followed by hardening at high extension ratios linked to the final extensibility of the network chains. The extrapolation of the softening regime to large strains $(1 / \lambda \rightarrow 0)$ gives the density of covalently crosslinked chains. ${ }^{60}$

As shown in Figures $\mathbf{5 b}$, below $T_{c}$, the gel demonstrates the classical response of a polymer network that is dominated by entropic elasticity, since the reduced stress vs. $1 / \lambda$ gives almost flat variation upon stretching. The extrapolated value of the reduced stress gives the elastic modulus $^{60,61}$ of the chemically-crosslinked network. No clear signature of entanglements in the tensile response (within the frequency investigated) is noticed. The modulus obtained in tension is in line with $G$ ' values obtained by linear rheology.

Conversely, at $60{ }^{\circ} \mathrm{C}$, the general shape of the tensile curve shown in Figure 5a, especially at the early stages of deformation, exhibits a linear stress-strain relation that significantly overestimates the purely entropic contribution of the network at $20^{\circ} \mathrm{C}$. This effect is clearly emphasized in Figure 5b, by an initial hardening, denoted as stage (1) whereas the PDMA network is supposed to be in coil state. This hardening can be ascribed to conformational changes that are harder to proceed and underlines the additional energetic contribution that arises from the phase-separation into hydrophobic concentrated PNIPAm domains. This early stage of deformation is followed by a softening regime (stage (2)) assigned to the deformation 
followed by disruption of collapsed domains that becomes readily effective above $\lambda=2$ and final strain hardening (stage (3) due to final extensibility of the network before failure.

As illustrated in Figures 5c, the formation of PNIPAm-rich domains increases the stiffness but also lead to an increase of the dissipative strain energy by the increase of hysteresis of the cyclic mechanical response. The dissipative mechanisms were noticed to be controlled by the stretching level and resulted in a residual strain just after unloading for applied strain levels beyond $50 \%$. Such a stretched-induced dissipation originates from the local rearrangements taking place in the phase-separated micro-domains, involving intrachain and inter-chain disruption of interacting NIPAm/NIPAm segments upon stretching. ${ }^{35,62}$ This mechanism enables the stress release and the energy dissipation, as sacrificial bonds. In contrast to covalent bond breaking, which is permanent, the phase-separation process involves reversible interactions such as $\mathrm{H}$-bonding and hydrophobic interactions that potentially may be reformed. As illustrated in Figure 5d, after a few minutes of rest, perfect recovery of both the strain and the mechanical behavior were noticed, suggesting that the dual-phase structure of the gel is perfectly reformed and the gel is able to self-heal in bulk for short period of rest. As expected from results obtained by linear rheology, the dissipative processes in GPD-N55 gels are weakly time-dependent. As already observed for the reverse topology ${ }^{33}$, here we demonstrated that the GPN-D55 gels also exhibit excellent fatigue resistance (see Supplementary Figure S5).

a)

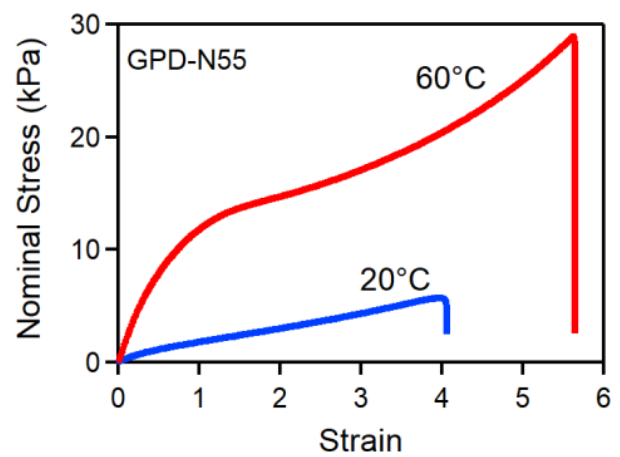

c) b)

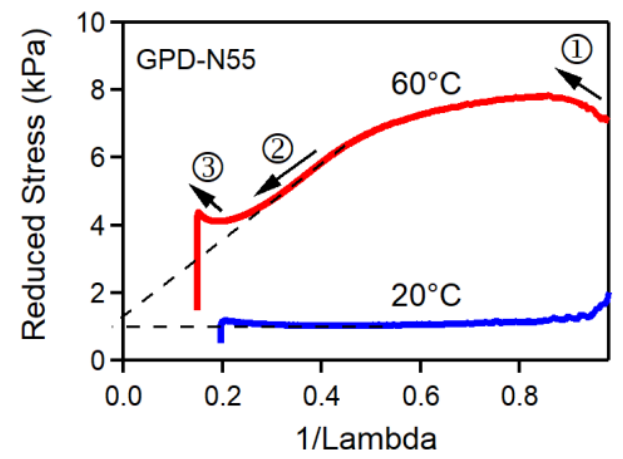

d) 

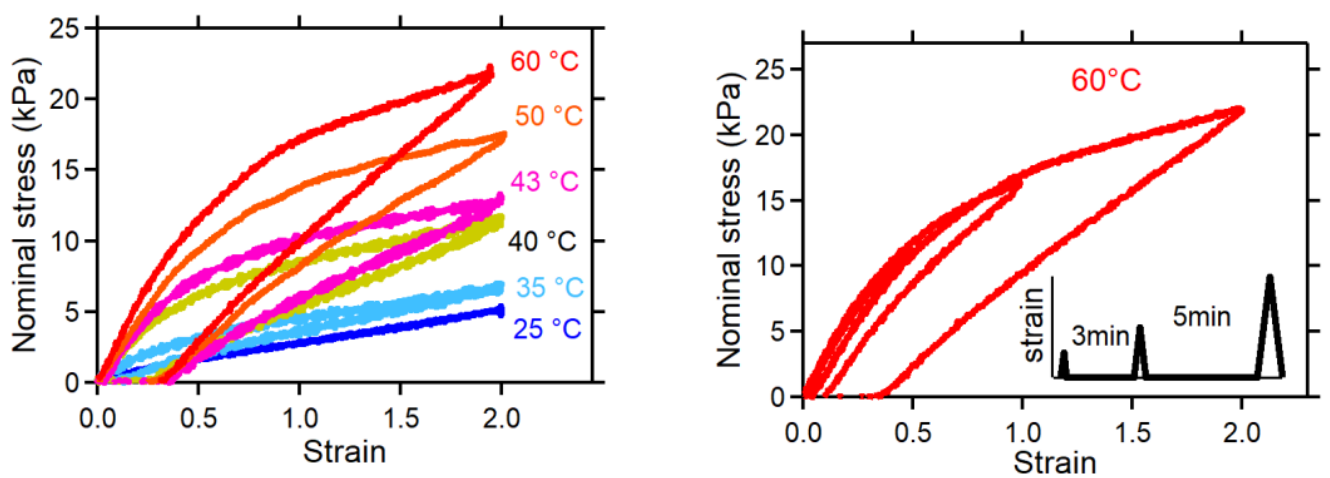

Figure 5. Large strain behavior of GPN-D gels. (a) Tensile stress-strain curves of GPD-N55 at 20 and $60{ }^{\circ} \mathrm{C}$. (b) Corresponding stress-strain curves plotted as the reduced stress versus $1 / \lambda$. This representation clarifies the deviations from classical rubber elasticity model displayed by the gel at $60{ }^{\circ} \mathrm{C}$ : initial hardening (stage (1)) is followed by a broad softening regime (stage (2)) before final strain hardening (stage (3)). The GPD-N behavior at $20{ }^{\circ} \mathrm{C}$ is given as a guideline and follows the theoretical rubberlike behavior. Interestingly, the extrapolation at $1 / \lambda \rightarrow 0$ of the softening $\left(60^{\circ} \mathrm{C}\right)$ coincided with the density of covalently crosslinked chains at $20{ }^{\circ} \mathrm{C}$. (c), Stress-strain cycles up to $200 \%$ of strain. Due to the thermoresponsive properties of PNIPAm, the gel exhibits a significant enhancement of dissipation by increasing temperature. (d), Strain and behavior recoveries were investigated by performing a series of loading/unloading cycles under different tensile stretches $(50 \%, 100 \%$ and $200 \%$ of strain) with the insertions of periods of rests in between ( 3 minutes and 5 minutes), see insert.

\section{Role of graft length and network on the mechanical reinforcement}

As already noticed for GPD-N55, while containing identical polymer weight composition (i.e. an overall polymer concentration of $16.3 \mathrm{wt} \%$ including $8.35 \mathrm{wt} \%$ of PNIPAm grafts), the significant tensile stiffening was observed at $60{ }^{\circ} \mathrm{C}$ compared to $20{ }^{\circ} \mathrm{C}$ for all the GPD-Nx series, as depicted in Figure 6 and Supplementary Figure S6. The elongation at break seems also to be enhanced at $60{ }^{\circ} \mathrm{C}$, except for GPD-N120 gels. As failure in this case systematically occurred in the clamps, this has to be taken with caution. This suggests that samples experienced damage in the jaws prior to the test. Specific focus on fracture properties will be given later in a dedicated section.

As shown in Figures 6a, the GPD-N120 gels exhibit the highest modulus at $60{ }^{\circ} \mathrm{C}$ that corresponds to a strong initial strain hardening, as illustrated in Figure 6c. Beyond a given strain value, a strong strain softening is observed which reflects the disruption of the physical fillers by the unravelling of PNIPAm grafts from globule conformation to stretched state. ${ }^{38}$ This suggests that long grafts allow to form well-defined stiff collapsed domains which lead to an efficient reinforcement. Interestingly, as shown in Figures 6c, all the curves seem to extrapolate for large elongation $(1 / \lambda \rightarrow 0)$ to a similar reduced stress of about $2 \mathrm{kPa}$, regardless 
of the length of the grafts. This is of the same order of magnitude as the response of the network at $20{ }^{\circ} \mathrm{C}$. Figures $\mathbf{6 b}$ and $\mathbf{6 d}$ show the large strain behavior for the GPD-N55 (y/z) series for comparison. By analogy with filled nanocomposites, the mechanical reinforcement was expected to be related to the fraction of hard domains. Surprisingly, as shown in Figure $\mathbf{6 b}$, the initial moduli are only weakly affected by the number of PNIPAm grafts. The extrapolations of the reduced strain at the late softening stage at $60{ }^{\circ} \mathrm{C}$ provide the signature of the PDMA network, since extrapolated values converge to the network signature at $20{ }^{\circ} \mathrm{C}$. Note that the strain at break is significantly increased by reducing the PDMA/PNIPAm ratio. For GPD-N55(31/69) gels, where the weight fraction of PDMA covalent network is about 5.2 $\mathrm{wt} \%$, i.e. close to the gel point, ${ }^{42}$ the strain at break can reach extremely high values (around $900 \%)$.

a)

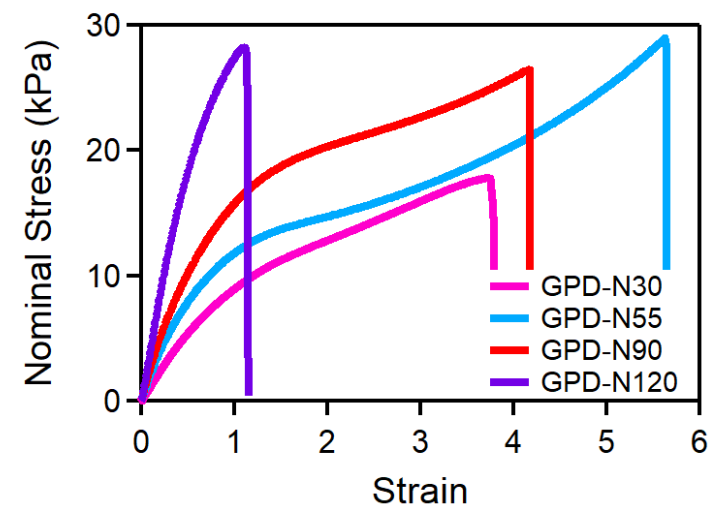

c)

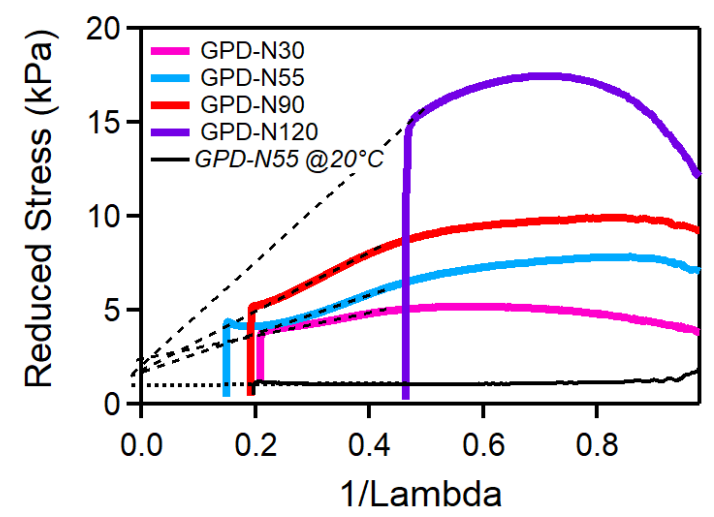

b)

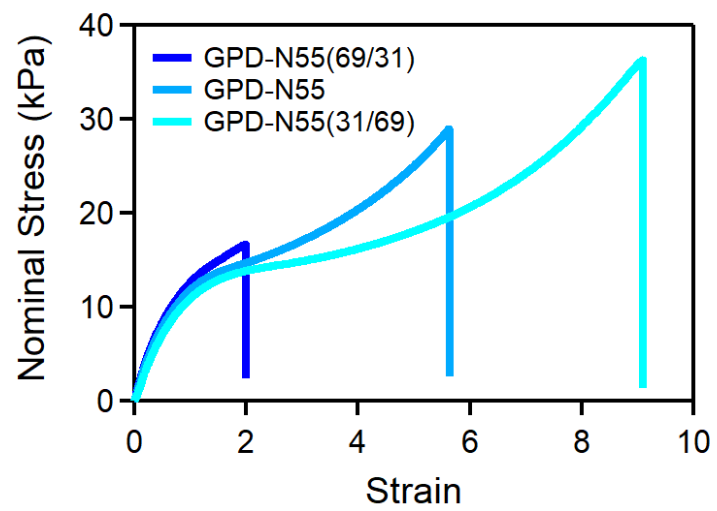

d)

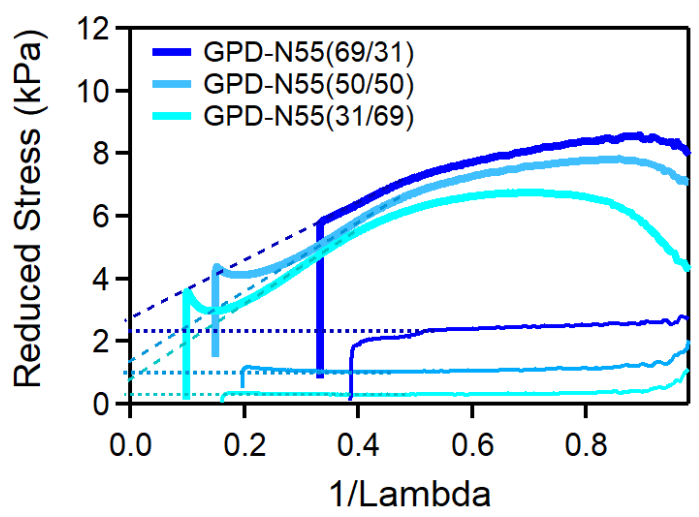

Figure 6. Large strain behavior of GPD-Nx and GPD-N55(y/z) hydrogels at $60{ }^{\circ} \mathrm{C}$. Tensile stress-strain curves of GPD-Nx (a) and GPD-N55(y-z) (b) gels. Corresponding stress-strain curves plotted as reduced stress as a function of $1 / \lambda$ for of GPD-Nx (c) and GPD-N55(y-z) 
(d). This representation highlights appreciable deviations from the predictions of the statistical theory. The GPD-Nx and GPD-N55(y/z) behaviours at $20{ }^{\circ} \mathrm{C}$ are given as a guideline and follow the theoretical rubberlike behavior.

Figure 7a reports the tensile moduli obtained at 20 and $60{ }^{\circ} \mathrm{C}$ for all the topologies investigated. As demonstrated in Figure 7a, it appears that initial elastic modulus is mainly controlled by the length of grafts, even in spite of the increasing volume fraction of grafted PNIPAm side chains. The GPD-N120 gels is characterized by the highest tensile modulus at $60{ }^{\circ} \mathrm{C}$ with $E$ around $44 \mathrm{kPa}$. By analogy to composite materials, we propose in Figure $7 \mathbf{b}$ an estimate of the stiffening ratio, as the ratio of the moduli at $60{ }^{\circ} \mathrm{C}$ (corresponding to the phaseseparated gels) and the moduli at $20{ }^{\circ} \mathrm{C}$ (reflecting the modulus of the network matrix). ${ }^{63}$ As expected, this parameter enlightens the remarkable thermo-reinforcement obtained for the GPD-N55(31/69) by considering the extremely weak signature of the PDMA network at the coil state. By heating above $T c$, the modulus of GPD-N55(31/69) is increased by about 35folds together with an enhancement of the stretch at break by 2 -folds. This is a quite remarkable result in terms of stimuli responsiveness as well as in the field of nanocomposite engineering. Conversely, the stiffening ratio attenuates the reinforcement effect for long grafts because the modulus at $20{ }^{\circ} \mathrm{C}$ is slightly higher.

a)

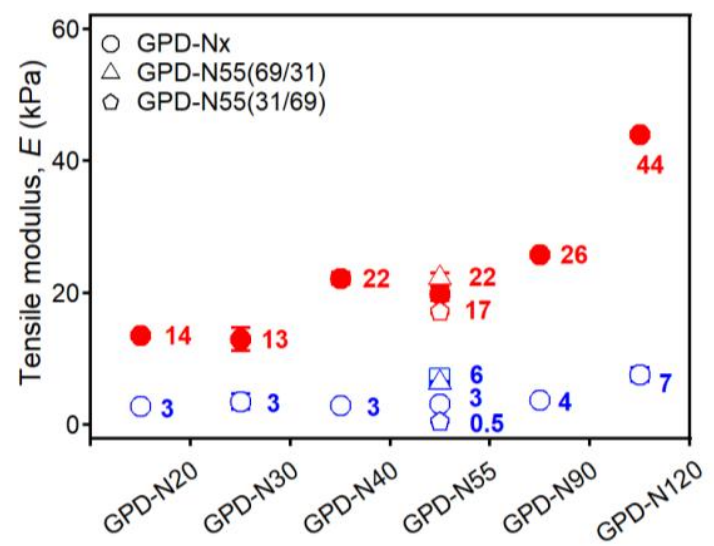

b)

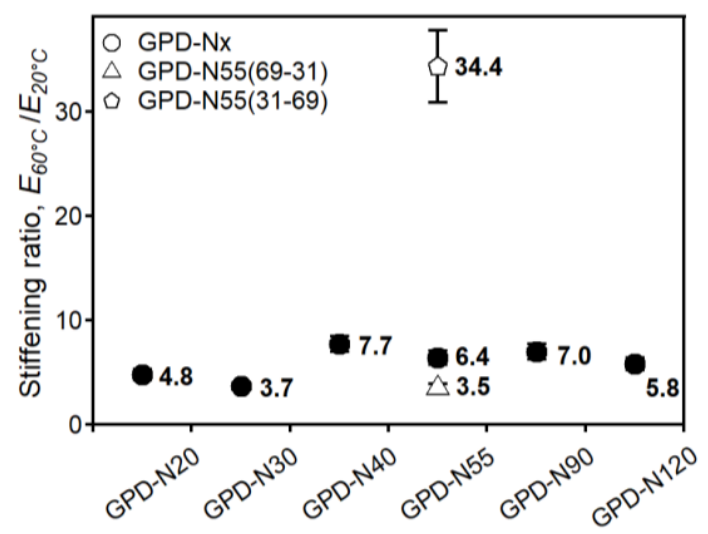

Figure 7. Summary of (a) the tensile modulus $E$ and (b) the stiffening ratio $E_{60^{\circ} \mathrm{C}} / E_{20^{\circ} \mathrm{C}}$ depending on both the PNIPAm side-chains average molar mass (GPD-Nx series) and the PDMA/PNIPAm composition of the network at both 20 and $60{ }^{\circ} \mathrm{C}$. All the tensile tests were performed at a $0.06 \mathrm{~s}^{-1}$ strain rate.

\section{Role of graft length on fracture toughening}


As already discussed, during tensile strength measurements, it is rather difficult to get rid out of the defect distribution or prevent premature failure in the jaws. To overcome this difficulty, fracture tests are performed on notched specimen with well-controlled notch lengths. As shown in Figure 8, the fracture energy, $G_{c}$, expressing the strain energy required to propagate the crack across the gels, is notably enhanced above the microphase separation. Interestingly, the length of PNIPAm grafts impacts strongly the fracture resistance of the gel. As shown in Figure 8, the fracture energies for the GPD-Nx series increase notably with the graft length. The GPD-N120 gels exhibit the highest fracture energy (around $150 \mathrm{~J} . \mathrm{m}^{-2}$ ), however neither the linear viscoelastic behavior, as the contribution of the dissipative component nor the work of extension given by the area under the tensile curve could anticipate this fracture behavior. This confirms that specific dissipative mechanisms takes place at the vicinity of the crack tip, where polymer chains experience high stretch ratios. This was also reported in opposite topologies, ${ }^{33}$ although they have almost analogous mechanical signatures in tension or linear rheology, gels may display very different fracture properties. The GPD-N(y/z) series seems to be more in line with expectations as fracture resistance is increased by increasing the amount of reinforcing agent, i.e. thermo-responsive moieties. Note that for all the gels, the crack was observed to propagate straightly; no crack bifurcation was noticed as it was systematically observed for PNIPAm networks with PDMA grafts. ${ }^{33}$ The length of the graft and its full unravelling from its globular conformation clearly appeared to be a key parameter for fracture toughening. As expected from Halperin and Zhulina's theoretical picture, ${ }^{38}$ the PNIPAm fillers act as a reservoir for stretch-induced dissipation: the longer the graft is, the more strain energy dissipation can proceed.

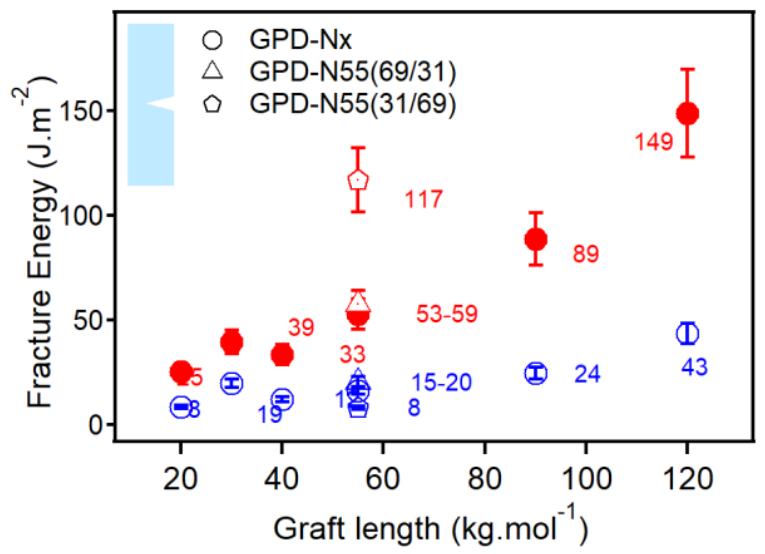

Figure 8. Fracture energies depending on both the PNIPAm graft length (GPD-Nx series) and the PDMA/PNIPAm composition at both $20^{\circ} \mathrm{C}$ (in blue) and $60{ }^{\circ} \mathrm{C}$ (in red). 

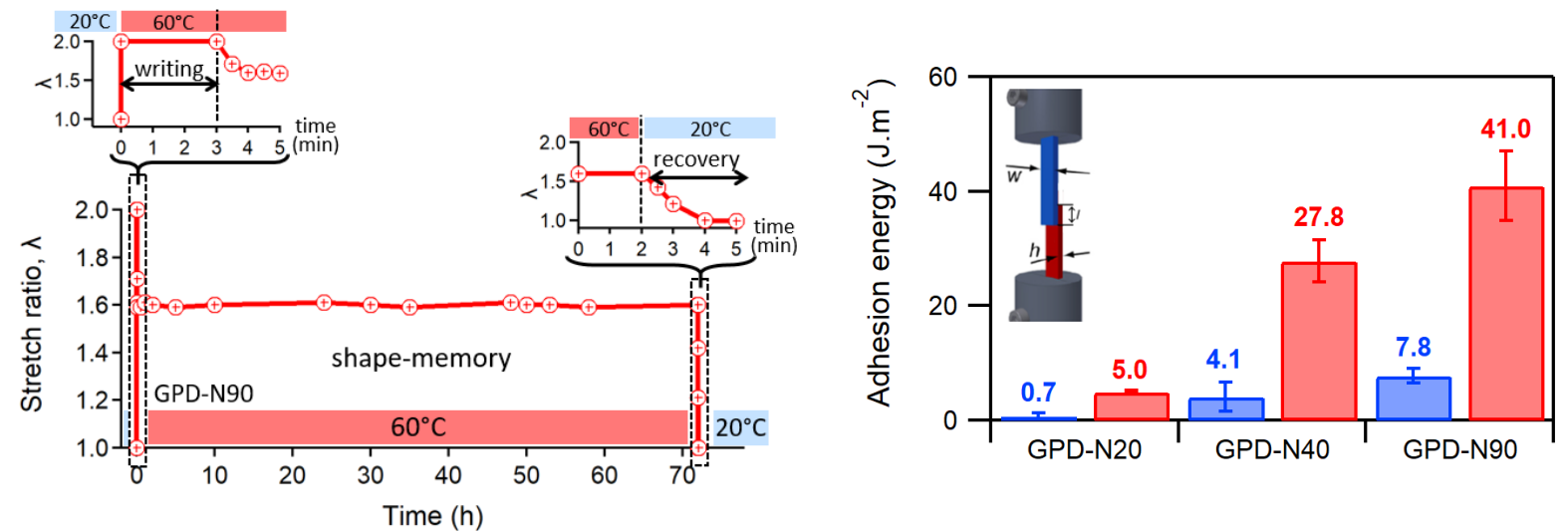

Figure 9. (a) Shape-memory curves of GPD-N90 gels with time. First, the gels were stretched to $\lambda=2$ at $20{ }^{\circ} \mathrm{C}$ prior to being heated at $60{ }^{\circ} \mathrm{C}$ to perform the "writing" procedure during 3 minutes. After writing for $3 \mathrm{~min}$, stress was released, and the residual stretched ratio was measured over time (3 days). Finally, the gels were cooled down to $20{ }^{\circ} \mathrm{C}$ to fulfil the "recovery" process. (b) Adhesion energies at 20 and $60{ }^{\circ} \mathrm{C}$. PNIPAm side-chains molar mass (GPD-Nx series). Insert: lap-shear test geometry.

Shape-memory and self-adhesion experiments were designed to probe the inter-chain bridging efficiency (in bulk or across the interface) together with the dissipative capabilities involved at large stretch ratio, as upon the peeling of bonded interfaces, for instance.

As illustrated for GPD-N90 in Figure 9a, the formation of phase-separated domains is also responsible for the shape memory effect. After "writing" a stretch ratio of $\lambda=2$ (strain of $100 \%$ ) at $60{ }^{\circ} \mathrm{C}$ during $3 \mathrm{~min}$, the stress was then removed. Partial retraction of the gel was noticed instantaneously, but gel held about $60 \%$ of residual strain suggesting that internal stresses due to the extension of the PDMA network are compensated by the formation of inter-chain micro-domains acting as physical cross-links. Note that the residual strain remained almost constant over time, even for days (72 hours). When the temperature was lowered to $20{ }^{\circ} \mathrm{C}$, the initial shape was quickly recovered since physical associations are suppressed for $T<T_{\mathrm{c}}$. As shown in Supplementary Figure S7, no significant effect of chain length was observed, suggesting an equivalent interchain bridging efficiency. Even for short grafts as in GPD-N20, gels were able to generate inter-chain aggregates to stabilize the PDMA network under a macroscopic strain of about $40 \%$.

In contrast, adhesion properties strongly depend on the length of the grafts, as shown in Figure 9b. Self-adhesion properties enable the exploration of PNIPAm grafts to bridge the interface between the two grafted hydrogels. Close contact was first established at $20{ }^{\circ} \mathrm{C}$, then the lap-joint assembly was maintained during 10 minutes under a constant pressure of $1 \mathrm{kPa}$ at a temperature $T\left(20\right.$ or $\left.60{ }^{\circ} \mathrm{C}\right)$. Figure $9 \mathbf{b}$ shows that adhesion energy increases significantly with graft length for both temperatures. At $20{ }^{\circ} \mathrm{C}$, the adhesion increases with the length of the dangling chain due to the combined effect of the efficiency to connect the two surfaces 
(by interdiffusion) and the increase of dissipative processes, as $G$ "' increases with the number of entanglements per dangling chain. At $60{ }^{\circ} \mathrm{C}$, a net increase of adhesion is observed due to the formation of phase-separated domains that toughen the interface between the two gels. Upon mechanical loading, the debonding systematically occurred by interfacial crack propagation. The adhesion energies are significantly increased at $60{ }^{\circ} \mathrm{C}$ compared to selfadhesion observed at $20{ }^{\circ} \mathrm{C}$. Adhesion energies involved are quite high (up to $40 \mathrm{~J}^{\mathrm{m}} \mathrm{m}^{-2}$ ) taking into account that adhesion is obtained without any covalent bonding. Note that this way adhesion can be tuned reversibly, adhesiveness can readily be switched-off by decreasing the temperature below $T_{c}$. Note that adhesive properties are qualitatively in line with fracture energies and confirm the crucial role of chain length for achieving efficient mechanical reinforcement in terms of fracture (in bulk or at interface) toughening. These results open interesting perspectives in the field ranging from underwater adhesion to thermally switchable adhesion.

\section{CONCLUSION}

Within the development of thermo-toughening gels that combine a hydrophilic polymer network (PDMA) with LCST polymer grafts (PNIPAm), the aim of this work was to explore new network topologies with varied PNIPAm graft lengths and number. Thermoresponsive grafts were expected to act as organic filler above a critical temperature, $T_{c}$. The free radical polymerization of DMA monomers with PNIPAm macromonomers and crosslinkers turns to be a simple method to design gels (in water) with rather well-controlled topologies and great versatility in compositions. By keeping constant the polymer weight fraction on both side of the phase transition (16.3 wt \%), thermo-responsive hydrogels demonstrated very different structure/properties relationships with perfectly reversible behaviors. Within the experimental range investigated, a remarkable combination of properties have been achieved: for sufficient long grafts, perfectly transparent phase-separated gels can be obtained which feature shape-memory properties, fast (thermo)switchable adhesion (as high as $40 \mathrm{~J} . \mathrm{m}^{-2}$ ) and increased fracture energies (up to $150 \mathrm{~J} . \mathrm{m}^{-2}$ ). These properties were observed to perform reversibly by thermal cycling.

As anticipated, the network topology is crucial, i.e. the overall polymer concentration as well as the weight fractions of PDMA and PNIPAm in hydrogels only partially control the 
gel properties, especially the mechanical reinforcement at large strains or other macroscopic behaviors such as optical properties. This work demonstrates it and refines our picture of reinforcing mechanisms:

1) The length of the PNIPAm grafts is the key parameter to enhance the mechanical stiffness and more specifically the fracture properties (bulk and adhesive). This study demonstrates that the longer the graft, the more effective the reinforcement. This is verified with the GPD-Nx series, for which longer grafts with larger distance between adjacent grafts, lead to smaller microphase separated domains but more efficient mechanical toughening. Above $T_{c}$, PNIPAm fillers act as a reservoir for stretch-induced dissipation: the longer the graft, the more strain energy dissipation can proceed.

2) To a lesser extent, the grafting density can also significantly improve the mechanical responses. Thus, as the hydrogel stiffening is closely related to the additional energetic contribution induced by PNIPAm phase-separated domains, as expected, increasing the PNIPAm/PDMA ratio enhances the reinforcement. This is observed for GPD-N(31/69) which display remarkable mechanical properties at phase-separated state (above $T_{c}$ ) with an increase of the elastic modulus and strain at break in a ratio by 35 and 2, respectively, compared to $20^{\circ} \mathrm{C}$.

It is noteworthy that linear viscoelastic properties do not enable one to anticipate on the fracture behaviours, since gels exhibit almost same dissipative contributions at $20{ }^{\circ} \mathrm{C}$ and $60{ }^{\circ} \mathrm{C}$ but significant enhanced fracture properties at $60{ }^{\circ} \mathrm{C}$. This confirms the importance of probing soft matter at large strains when large scale rearrangements take place and produce efficient dissipative processes.

From a prospective viewpoint, the design of perfectly transparent phase-separated gels, as demonstrated here for GPD-N90 and GPD-N120, opens on applicative fields for which transparency together with shape-memory properties ${ }^{64}$ or responsive hydrophobicity and adhesion are desirable. ${ }^{65-67}$ 


\section{Supporting Information}

\section{Supplementary Experimental Data}

Supplementary Figure S1. Geometries used for large strain mechanical testing.

Supplementary Figure S2. PNIPAm macromonomers characteristics determined by Size Exclusion Chromatography

Supplementary Figure S3. Endotherms obtained by Differential Scanning Calorimetry for GPD-Nx series. Comparison of phase-separation temperature, $T_{c}$ determined by turbidimetry (UV-vis), Differential Scanning Calorimetry (DSC) and linear rheology ( $G$ ' and $\tan \delta$ )

Supplementary Figure S4. Dynamic shear elastic (or storage) modulus $G$ ' (filled symbols) and loss modulus $G$ '” (open symbols) for a homo-PDMA gel.

Supplementary Figure S5. Fatigue test in tensile mode for GPD-N55 at $60{ }^{\circ} \mathrm{C}, 100$ consecutive cycles were applied at $60{ }^{\circ} \mathrm{C}$.

Supplementary Figure S6. Large strain behavior of GPD-Nx gels. (a) Tensile stress-strain curves of GPD-Nx at $20^{\circ} \mathrm{C}$ and $60{ }^{\circ} \mathrm{C}$. (b) Corresponding stress-strain curves plotted as reduced stress as a function of $1 / \lambda$.

Supplementary Figure S7. Shape-memory curves of GPD-Nx series.

\section{AUTHOR INFORMATION}

Corresponding Author

*E-mail alba.marcellan@espci.fr

\section{ACKNOWLEDGMENTS}

Ph.D. scholarship of C.M. was provided by the Ph.D. school "Chemistry and Physics of Materials" of Sorbonne University (ED 397, SU, Paris, France). The authors would like to thank Prof. Rémi Carminati for fruitful discussions on optical properties, Guylaine Ducouret from SIMM for technical advice on performing rheological measurements and Annie Brûlet from LLB for her contribution on SANS measurements.

\section{NOTES}

The authors declare no competing financial interest. 


\section{REFERENCES}

1. P. G. De Gennes, Scaling Concepts in Polymer Physics, Cornell University Press, Ithaca, 1979.

2. M. Shibayama and T. Tanaka, Adv. Polym. Sci., 1993, 109, 1-62.

3. T. Tanaka, Physical Review Letters, 1978, 40, 820-823.

4. A. Onuki, Adv. Polym. Sci., 1993, 109, 63-121.

5. K. Dušek and D. Patterson, Journal of Polymer Science Part A-2: Polymer Physics, 1968, 6, 1209-1216.

6. A. Halperin, M. Kroger and F. M. Winnik, Angew. Chem.-Int. Edit., 2015, 54, 1534215367.

7. M. Heskins and J. E. Guillet, Journal of Macromolecular Science: Part A - Chemistry, 1968, 2, 1441-1455.

8. Q. Zhang, C. Weber, U. S. Schubert and R. Hoogenboom, Materials Horizons, 2017, 4, 109-116.

9. $\quad$ P. Calvert, Adv. Mater., 2009, 21, 743-756.

10. D. J. Beebe, J. S. Moore, J. M. Bauer, Q. Yu, R. H. Liu, C. Devadoss and B. H. Jo, Nature, 2000, 404, 588-+.

11. L. Dong, A. K. Agarwal, D. J. Beebe and H. Jiang, Nature, 2006, 442, 551-554.

12. D. C. Coughlan, F. P. Quilty and O. I. Corrigan, Journal of Controlled Release, 2004, 98, 97-114.

13. Y. Li, G. H. Gao and D. S. Lee, Advanced Healthcare Materials, 2013, 2, 388-417.

14. T. Dvir, B. P. Timko, D. S. Kohane and R. Langer, Nat. Nanotechnol., 2011, 6, 13-22.

15. L. Klouda and A. G. Mikos, European Journal of Pharmaceutics and Biopharmaceutics, 2008, 68, 34-45.

16. P. Schattling, F. D. Jochum and P. Theato, Chemical Communications, 2011, 47, 8859-8861.

17. X. H. Wang, X. P. Qiu and C. Wu, Macromolecules, 1998, 31, 2972-2976.

18. C. W. Peak, J. J. Wilker and G. Schmidt, Colloid Polym. Sci., 2013, 291, 2031-2047.

19. X. Zhao, Soft Matter, 2014, 10, 672-687.

20. J. P. Gong, Y. Katsuyama, T. Kurokawa and Y. Osada, Adv. Mater., 2003, 15, 1155-+.

21. J. Y. Sun, X. H. Zhao, W. R. K. Illeperuma, O. Chaudhuri, K. H. Oh, D. J. Mooney, J. J. Vlassak and Z. G. Suo, Nature, 2012, 489, 133-136.

22. T. L. Sun, T. Kurokawa, S. Kuroda, A. Bin Ihsan, T. Akasaki, K. Sato, M. A. Haque, T. Nakajima and J. P. Gong, Nature Materials, 2013, 12, 932-937.

23. S. Abdurrahmanoglu, V. Can and O. Okay, Polymer, 2009, 50, 5449-5455.

24. S. Kondo, T. Hiroi, Y. Han, T.-H. Kim, M. Shibayama, U. Chung and T. Sakai, Adv. Mater., 2015, 27, 7407-7411.

25. K. Sato, T. Nakajima, T. Hisamatsu, T. Nonoyama, T. Kurokawa and J. P. Gong, Adv. Mater., 2015, 27, 6990-+.

26. K. Haraguchi, T. Takehisa and S. Fan, Macromolecules, 2002, 35, 10162-10171.

27. C. J. Wu, J. J. Wilker and G. Schmidt, Macromol. Biosci., 2013, 13, 59-66.

28. S. Rose, A. Prevoteau, P. Elziere, D. Hourdet, A. Marcellan and L. Leibler, Nature, 2014, 505, 382-385.

29. S. Rose, A. Dizeux, T. Narita, D. Hourdet and A. Marcellan, Macromolecules, 2013, 46, 4095-4104.

30. L.-W. Xia, R. Xie, X.-J. Ju, W. Wang, Q. Chen and L.-Y. Chu, Nature Communications, 2013, 4, 2226.

31. D. E. Apostolides, T. Sakai and C. S. Patrickios, Macromolecules, 2017, 50, 2155 2164. 
32. F. Wang and R. A. Weiss, Macromolecules, 2018, 51, 7386-7395.

33. H. Guo, N. Sanson, D. Hourdet and A. Marcellan, Adv. Mater., 2016, 28, 5857-5864.

34. H. Kamata, Y. Akagi, Y. Kayasuga-Kariya, U.-i. Chung and T. Sakai, Science, 2014, 343, 873-875.

35. H. Guo, C. Mussault, A. Brulet, A. Marcellan, D. Hourdet and N. Sanson, Macromolecules, 2016, 49, 4295-4306.

36. H. Guo, N. Sanson, A. Marcellan and D. Hourdet, Macromolecules, 2016, 49, 95689577.

37. K. Haraguchi, K. Uyama and H. Tanimoto, Macromolecular rapid communications, 2011, 32, 1253-1258.

38. A. Halperin and E. B. Zhulina, Europhysics Letters, 1991, 15, 417-421.

39. H. Guo, A. Brûlet, P. R. Rajamohanan, A. Marcellan, N. Sanson and D. Hourdet, Polymer, 2015, 60, 164-175.

40. H. W. Greensmith, Journal of Applied Polymer Science, 1963, 7, 993-1002.

41. K. Kendall, J. Adhes., 1975, 7, 137-140.

42. L. Carlsson, S. Rose, D. Hourdet and A. Marcellan, Soft Matter, 2010, 6, 3619-3631.

43. S. Rose, A. Marcellan, T. Narita, F. Boue, F. Cousin and D. Hourdet, Soft Matter, 2015, DOI: 10.1039/c5sm01121c.

44. X. Wang, X. Qiu and C. Wu, Macromolecules, 1998, 31, 2972-2976.

45. M. Heskins and J. E. Guillet, J. Macromol. Sci.-Chem., 1968, 2, 1441-1455.

46. F. Afroze, E. Nies and H. Berghmans, Journal of Molecular Structure, 2000, 554, 5568.

47. Y. Okada and F. Tanaka, Macromolecules, 2005, 38, 4465-4471.

48. M. Shibayama, M. Morimoto and S. Nomura, Macromolecules, 1994, 27, 5060-5066.

49. A. Y. Grosberg and S. K. Nechaev, Macromolecules, 1991, 24, 2789-2793.

50. S. Hirotsu, Macromolecules, 1990, 23, 903-905.

51. S. Hirotsu, Macromolecules, 2004, 37, 3415-3424.

52. L. R. G. Treloar, Transactions of the Faraday Society, 1942, 38, 0293-0298.

53. P. J. Flory and J. Rehner, J. Chem. Phys., 1943, 11, 512-520.

54. H. L. Needles and R. E. Whitfield, Journal of Organic Chemistry, 1964, 29, 3632-\&.

55. J. G. Curro and P. Pincus, Macromolecules, 1983, 16, 559-562.

56. L. H. Cai, T. E. Kodger, R. E. Guerra, A. F. Pegoraro, M. Rubinstein and D. A. Weitz, Adv. Mater., 2015, 27, 5132-5140.

57. M. Shibayama, Polymer Journal, 2011, 43, 18-34.

58. J. Bastide and L. Leibler, Macromolecules, 1988, 21, 2647-2649.

59. M. Mooney, Journal of Applied Physics, 1940, 11, 582.

60. L. Mullins, Journal of Applied Polymer Science, 1959, 2, 257-263.

61. In fact, extrapolation gives the shear elastic modulus. Note that sssuming the volume change upon stretching being negligible, as expected from rubber elasticity, the relation

between the tensile modulus, $\mathrm{E}$ and the shear modulus, $G$ is given by: $E=2(1+v) G \cong$ $3 G$ with $v$, the Poisson's ratio. Pure incompressible behavior gives $v \rightarrow 0.5$.

62. T. Takigawa, T. Ikeda, Y. Takakura and T. Masuda, The Journal of Chemical Physics, 2002, 117, 7306-7312.

63. E. Guth, Journal of Applied Physics, 1945, 16, 20-25.

64. H. Xiaobo, Z. Daixuan and S. S. S., Adv. Mater., 2018, 30, 1707461.

65. T. Okano, N. Yamada, M. Okuhara, H. Sakai and Y. Sakurai, Biomaterials, 1995, 16, 297-303.

66. C. D. H. Alarcon, S. Pennadam and C. Alexander, Chemical Society Reviews, 2005, 34, 276-285. 
67. E. G. Kelley, J. N. L. Albert, M. O. Sullivan and T. H. Epps, Chemical Society Reviews, 2013, 42, 7057-7071. 


\section{SUPPLEMENTARY INFORMATION}

\section{Supplementary Experimental Section}

Synthesis of PNIPAm macromonomers. The synthesis involved a two-step process:

\section{1) Synthesis of Amino-Terminated Telomers}

The synthesis of functional chains was achieved by radical polymerization using 2Amino-ethane-thiol hydrochloride (AET.HCl, $\geq 97 \%$, Aldrich) as chain transfer agent. This telomerization allows controlling the end group of the polymer as well as its molar mass. In a three necked flask, $100 \mathrm{mmol}$ of PNIPAm monomers was dissolved in $100 \mathrm{~mL}$ of water and the solution was deoxygenated for $1 \mathrm{~h}$ with nitrogen bubbling. The redox initiators, KPS (1.0 $\mathrm{mmol})$ and AET. $\mathrm{HCl}(2.0 \mathrm{mmol})$, were separately dissolved in $10 \mathrm{~mL}$ of water and deoxygenated for $30 \mathrm{~min}$ before adding to the monomer solution. The reaction was allowed to proceed in an ice bath, in order to avoid the phase separation of the reaction medium in the case of PNIPA. SEC was applied to monitor the polymerization process. After $4 \mathrm{~h}$, no monomer was left according to SEC, and an appropriate amount of sodium hydroxide was added to neutralize the hydrochloride ions and the polymer was recovered by dialysis against pure water (membrane cut-off $=3.5 \mathrm{kDa}$ ) for one week and freeze-dried. The telomers were obtained with a yield of $70 \mathrm{wt} \%$.

\section{2) Synthesis of linear macromonomers}

Typically for the PNIPAm macromonomer, $6.0 \mathrm{~g}$ of amino-terminated telomers (about 0.26 $\mathrm{mmol})$ and $0.29 \mathrm{~g}$ of AA (4 mmol) were initially dissolved in $50 \mathrm{~mL}$ of NMP at room temperature. After dissolution, $0.8 \mathrm{~g}$ of DCCI $(4 \mathrm{mmol})$, initially dissolved in a minimum of NMP, was introduced rapidly and the reaction was allowed to proceed overnight under stirring at room temperature. After dilution with $100 \mathrm{~mL}$ of water, the polymer was purified by dialysis against pure water (membrane cut-off $=3.5 \mathrm{kDa}$ ) for one week, and the aqueous solutions were filtered and freeze dried. The reaction was almost quantitative and the PNIPAm macromonomer was obtained with a yield of $95 \mathrm{wt} \%$. 
(a)

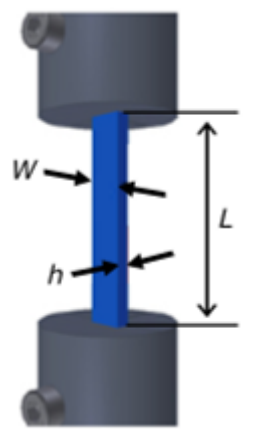

(b)

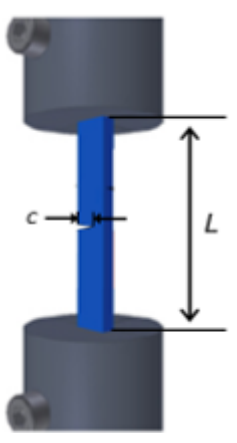

(c)

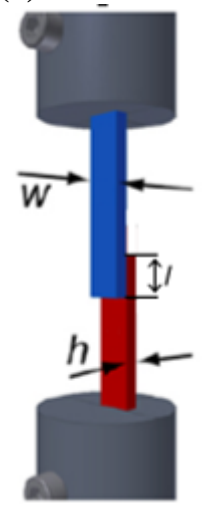

Supplementary Figure S1. Geometries used for large strain mechanical testing (a) Uni-axial tensile test geometry; (b) Fracture test geometry with c being the initial crack length; (c) Lapjoint geometry.

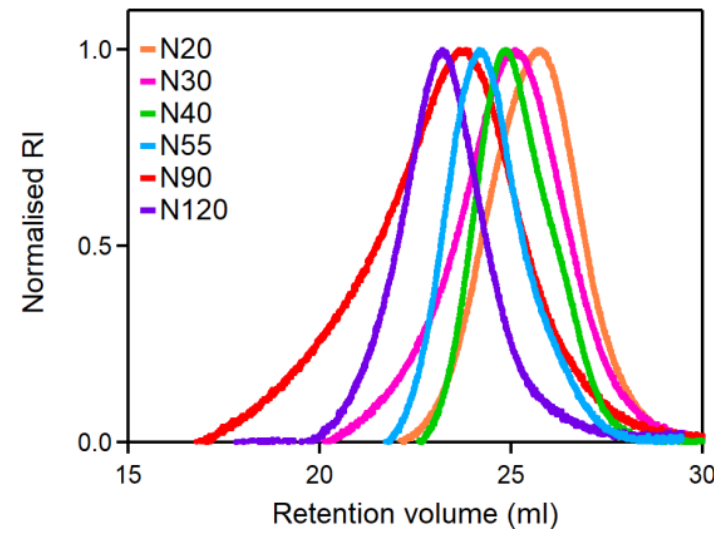

\begin{tabular}{ccccc}
\hline Macromonomer & $\begin{array}{c}\mathrm{M}_{\mathrm{n}} \\
(\mathrm{kg} / \mathrm{mol})\end{array}$ & $\begin{array}{c}\mathrm{M}_{\mathrm{w}} \\
(\mathrm{kg} / \mathrm{mol})\end{array}$ & DPn & Đ \\
\hline PNIPAm-N20 & 18.9 & 22.4 & 170 & 1.2 \\
PNIPAm-N30 & 32.9 & 48.2 & 290 & 1.5 \\
PNIPAm-N40 & 39.3 & 52.8 & 350 & 1.3 \\
PNIPAm-N55 & 54.9 & 73.9 & 485 & 1.4 \\
PNIPAm-N90 & 87.2 & 123.3 & 770 & 2.4 \\
PNIPAm-N120 & 121.0 & 177.2 & 1070 & 1.5 \\
\hline
\end{tabular}

Supplementary Figure S2. PNIPAm macromonomers characteristics determined by Size Exclusion Chromatography
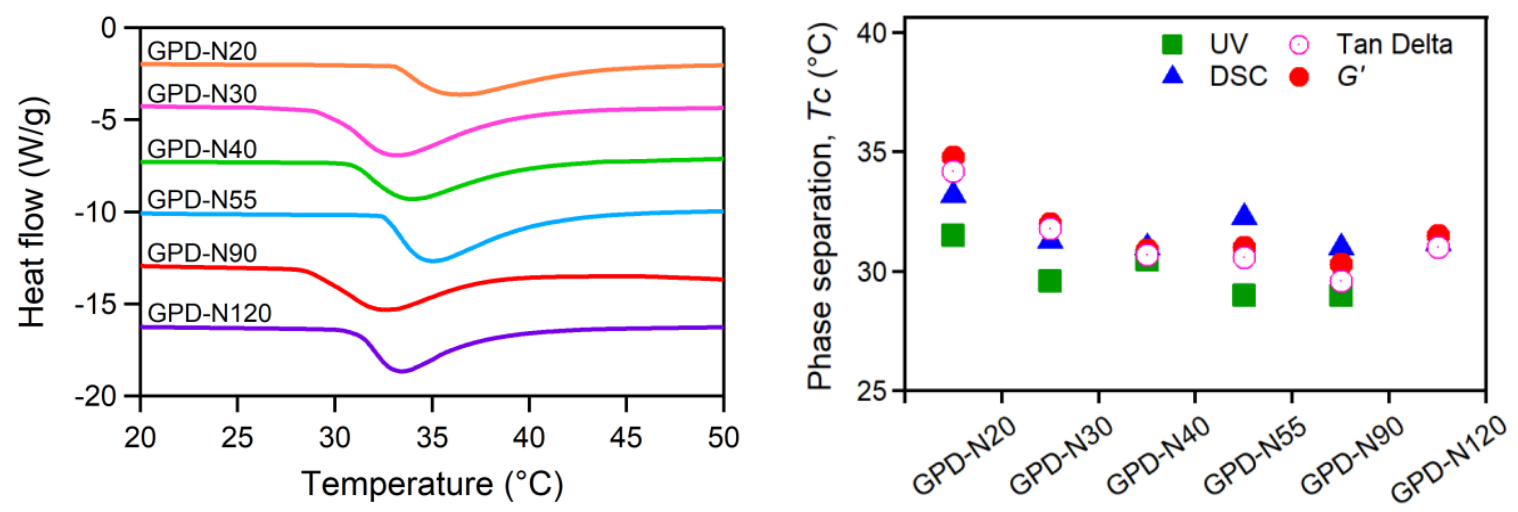

Supplementary Figure S3. a) Endotherms obtained by differential scanning calorimetry upon heating reflects the phase-transition undergone by PNIPAm side-chains forming microdomains. b) Comparison of phase-separation temperature, $T_{c}$ determined by turbidimetry (UV-vis), Differential Scanning Calorimetry (DSC) and linear rheology ( $G^{\prime}$ and $\tan \delta$ ) 


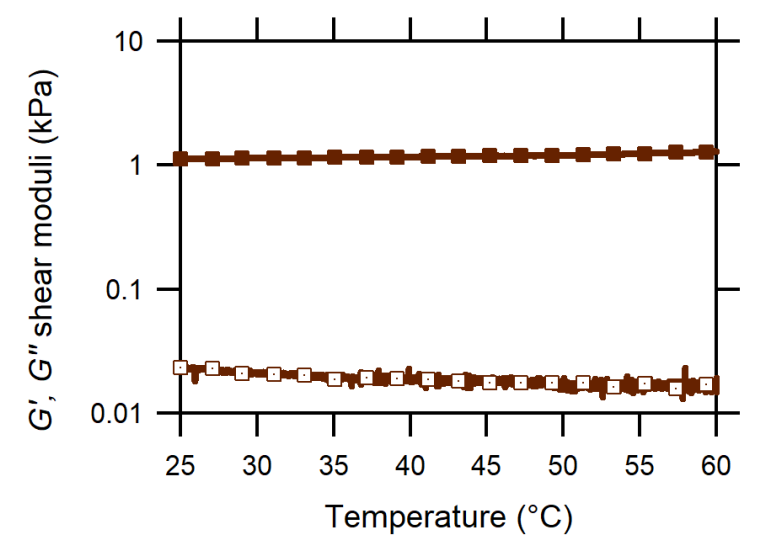

Supplementary Figure S4. Dynamic shear elastic (or storage) modulus $G$ ' (filled symbols) and loss modulus $G$ '” (open symbols) for a homo-PDMA gel.

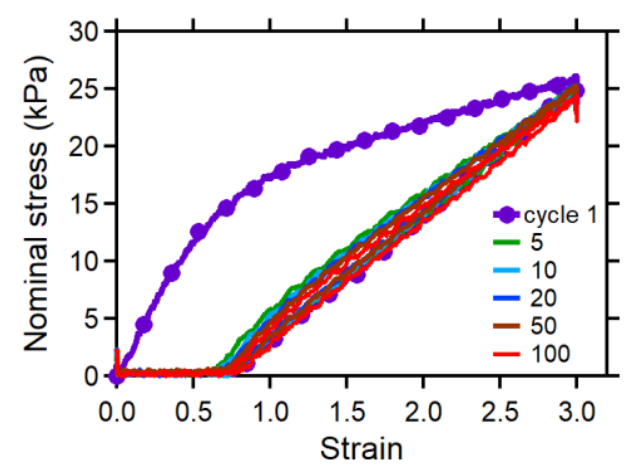

Supplementary Figure S5. Fatigue test in tensile mode for GPD-N55 at $60{ }^{\circ} \mathrm{C}, 100$ consecutive cycles were applied at $60^{\circ} \mathrm{C}$. Note that hundreds of consecutive cycles present almost closed-loops and an overlapping mechanical response, with no lost in stiffness or creeping phenomena over time 
a)
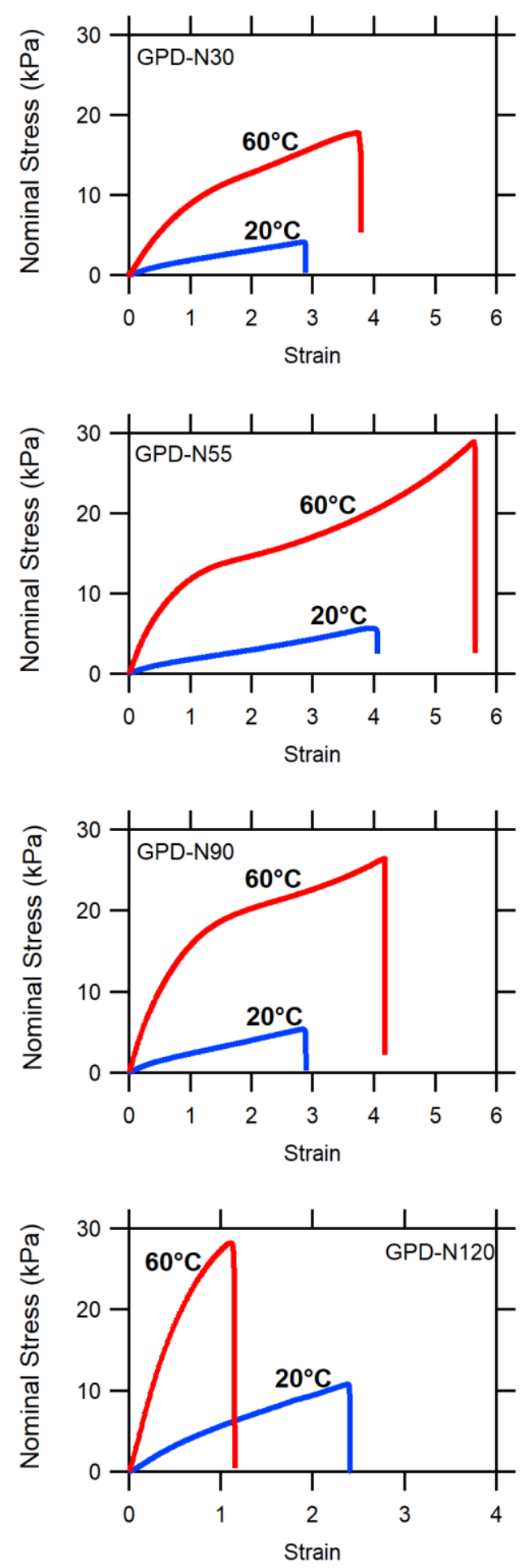

b)
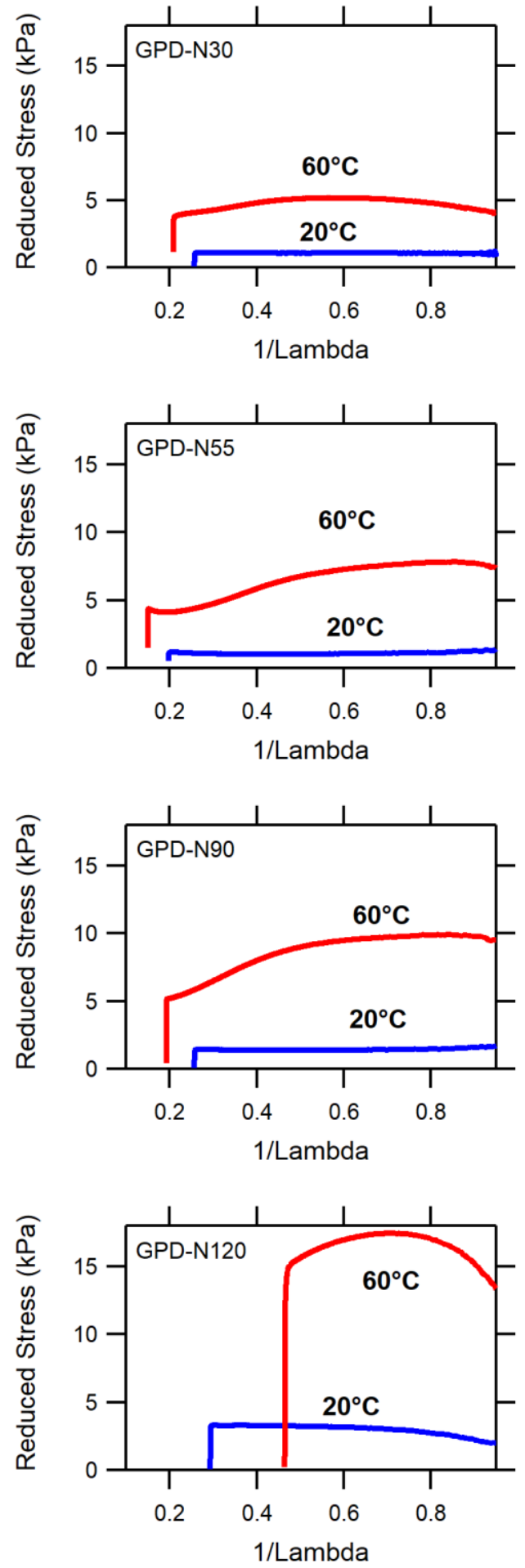

Supplementary Figure S6. Large strain behavior of GPD-Nx gels. (a) Tensile stress-strain curves of GPD-Nx at $20^{\circ} \mathrm{C}$ and $60^{\circ} \mathrm{C}$. (b) Corresponding stress-strain curves plotted as reduced stress as a function of $1 / \lambda$. This representation enhances the deviations from the classical rubber elasticity model displayed by the gel at $60{ }^{\circ} \mathrm{C}$ : a strong hardening is observed at the early stages of deformation followed by softening above $50 \%$ of strain. The GPD-Nx behavior at $20{ }^{\circ} \mathrm{C}$ is given as a guideline and follows the theoretical rubberlike behavior. 
(a)

(b)

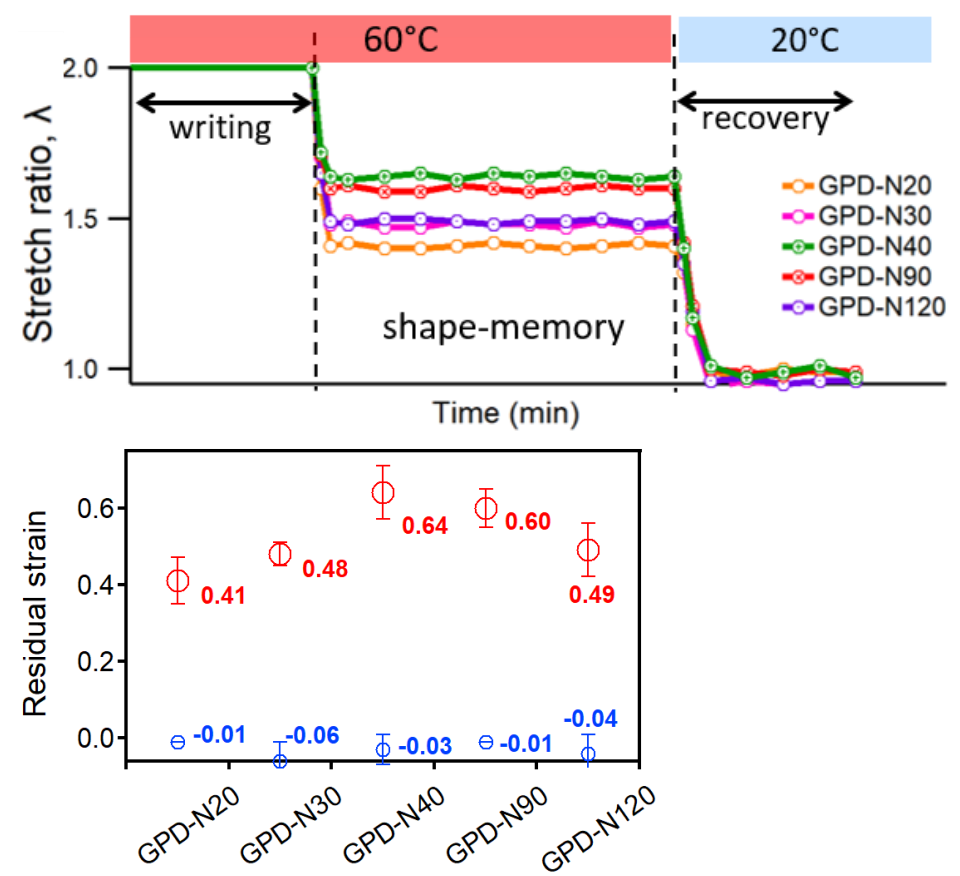

Supplementary Figure S7. Shape-memory curves of GPD-Nx series. (a) First, the gels were stretched to $\lambda=2$ at $20{ }^{\circ} \mathrm{C}$ prior to be heated at $60{ }^{\circ} \mathrm{C}$ to perform the "writing" procedure during 3 minutes. After writing for 3 min, stress was released, and the residual stretched ratio was measured over time. Finally, the gels were cool down at $20^{\circ} \mathrm{C}$ to fulfil the "recovery" process. (b) Summary of the residual strain at 60 and $20{ }^{\circ} \mathrm{C}$ after the "writing" and the "recovery" processes, respectively. 